\title{
Endogenous Calcitonin Gene-Related Peptide Deficiency Exacerbates Postoperative Lymphedema by Suppressing Lymphatic Capillary Formation and M2 Macrophage Accumulation
}

Shuhei Matsui, ${ }^{* \dagger}$ Megumu Tanaka, ${ }^{*}$ Akiko Kamiyoshi, ${ }^{* \ddagger}$ Takayuki Sakurai, ${ }^{*}$ Yuka Ichikawa-Shindo, ${ }^{*}$ Hisaka Kawate, ${ }^{*}$ Kun Dai, ${ }^{*}$ Nanqi Cui, ${ }^{*}$ Yangxuan Wei, ${ }^{*}$ Masaaki Tanaka, ${ }^{\S}$ Shinji Kakihara, ${ }^{\circledR}$ Keisei Nakamura, ${ }^{*}$ Akihiro Yamauchi, ${ }^{*}$ Kumiko Ishida, ${ }^{\dagger}$ Satoshi Tanaka, ${ }^{\dagger}$ Mikito Kawamata, ${ }^{\dagger}$ and Takayuki Shindo**

From the Departments of Cardiovascular Research, $*$ Anesthesiology, ${ }^{\dagger}$ and Ophthalmology, ${ }^{\S}$ Shinshu University School of Medicine, Matsumoto, Nagano, Japan; and the Department of Life Innovation, ${ }^{\ddagger}$ Institute for Biomedical Sciences, Interdisciplinary Cluster for Cutting Edge Research, Shinshu University, Matsumoto, Nagano, Japan

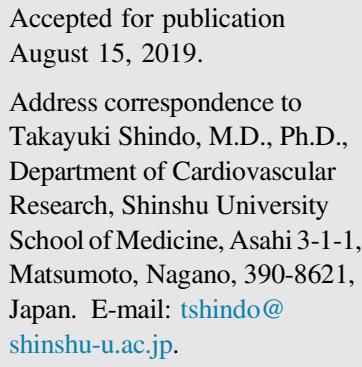

\begin{abstract}
Lymphedema is a chronic condition caused by disruption of lymphatic vessels, which often occurs after invasive surgery. Calcitonin gene-related peptide (CGRP) is a 37-amino acid peptide produced by alternative splicing of the primary transcript of the calcitonin/CGRP gene (Calca). CGRP was initially identified as a neuropeptide released primarily from sensory nerves and involved in regulating pathophysiological nociceptive pain. However, recent studies have shown CGRP is also released from a variety of other cells and possesses multiple functions. In this study, CGRP knockout (-/-) mice were used to show the actions of endogenous CGRP in postoperative lymphedema. After generating a mouse postoperative tail lymphedema model, the edema was observed to be more severe in $\mathrm{CGRP}^{-/-}$mice than in wild-type mice. Numbers of lymphatic vessel endothelial hyaluronan receptor 1 (LYVE-1)-positive lymphatic capillaries were decreased and lymphatic capillary formation-related factors were down-regulated in CGRP ${ }^{-/-}$mice. In addition, accumulation of $\mathrm{M} 2$ but not M1 macrophages was selectively reduced in the edematous tissue of CGRP ${ }^{-/-}$ mice. Selective depletion of M2 macrophages decreased lymphatic capillary formation and worsened lymphedema in wild-type mice but not $\mathrm{CGRP}^{-/-}$mice, where numbers of M2 macrophages were already diminished. These findings suggest that endogenous CGRP acts to ameliorate postoperative lymphedema by enhancing lymphatic capillary formation and that M2 macrophages play critical roles. CGRP may be a useful therapeutic target for the treatment of postoperative lymphedema. (Am J Pathol 2019, 189: 2487-2502; https://doi.org/10.1016/j.ajpath.2019.08.011)
\end{abstract}

Lymphedema is a chronic condition caused by disruption of lymphatic vessels, often after lymph node resection and radiation therapy. In extreme cases, supermicrosurgical lymphaticovenular anastomosis is applied, ${ }^{1,2}$ but the operation is not curative, and continued therapy remains necessary. A better understanding of the molecular and cellular mechanisms involved in the regulation of lymphatic vessels will bring new insight to lymphedema treatment.

Calcitonin gene-related peptide (CGRP) is a 37-amino acid peptide produced through alternative splicing of the primary transcript of the calcitonin/CGRP gene (Calca) ${ }^{3}$

\footnotetext{
Supported by Grants-in-Aid for Scientific Research (KAKENHI); Core Research for Evolutionary Science and Technology (CREST) of the Japan Agency for Medical Research and Development (AMED); and research grants from Bristol-Myers Squibb Company, Japan Foundation for Applied Enzymology, the Naito Foundation, the Public Foundation of Chubu Science and Technology Center, Yamaguchi Endocrine Research Foundation, Hoyu Science Foundation, Takahashi Industrial and Economic Research Foundation, Akaeda Medical Research Foundation, Shinshu Public Utility Foundation for Promotion of Medial Sciences, and Japan Heart Foundation (all to T.Sh.)

Disclosures: None declared.
} 
Table 1 Primers and Probes Used for Real-Time Quantitative RT-PCR

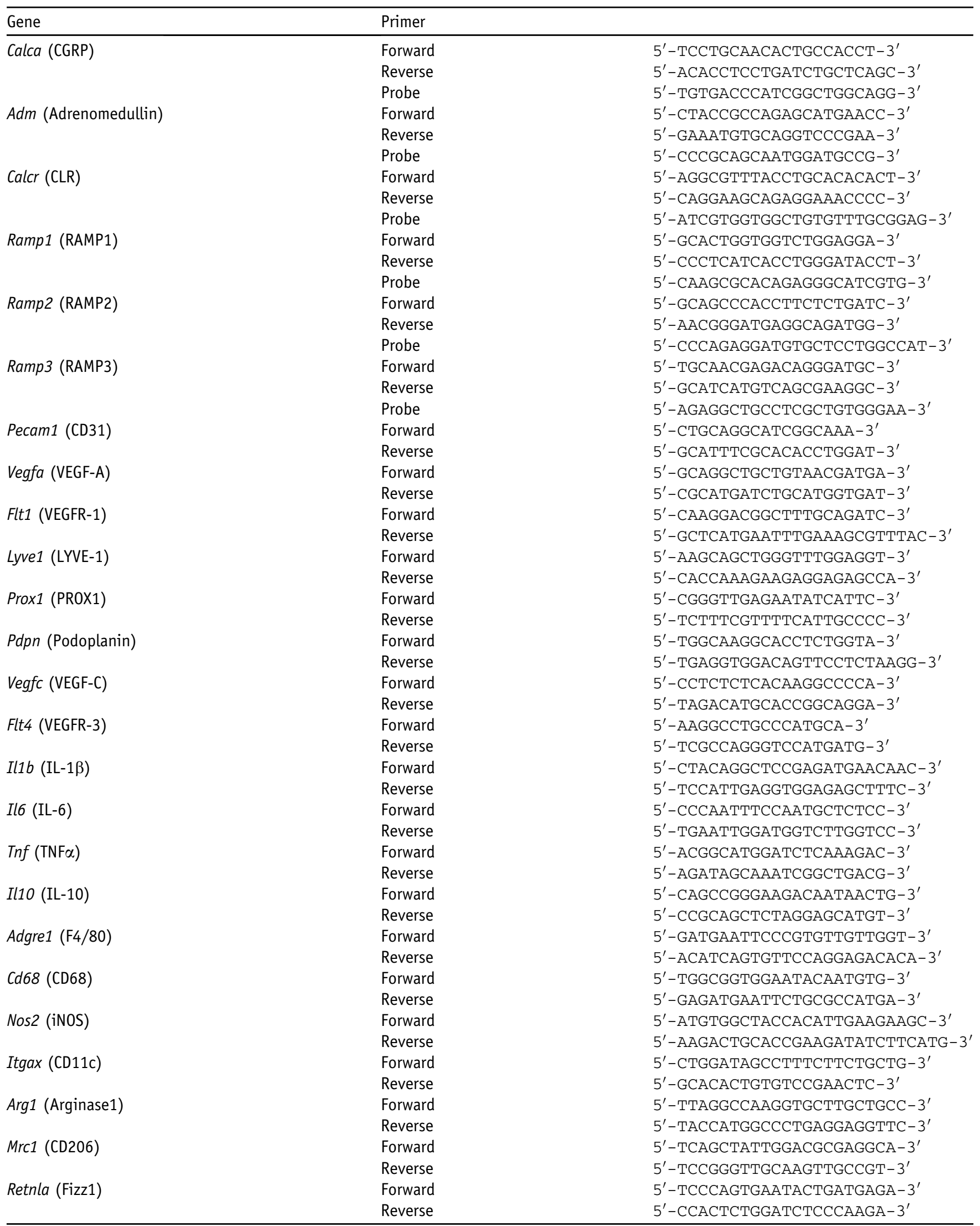

CGRP, calcitonin gene-related peptide; CLR, calcitonin receptor-like receptor; iNOS, inducible nitric oxide synthase; LYVE-1, lymphatic vessel endothelial hyaluronan receptor 1; RAMP, receptor activity-modifying protein; TNF, tumor necrosis factor; VEGF, vascular endothelial growth factor; VEGFR, vascular endothelial growth factor receptor. 
A

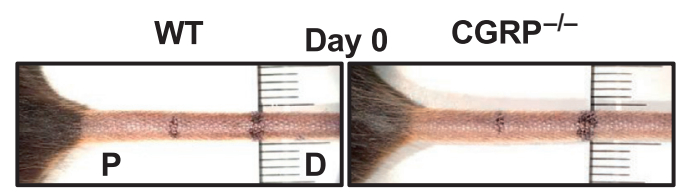

Day 2

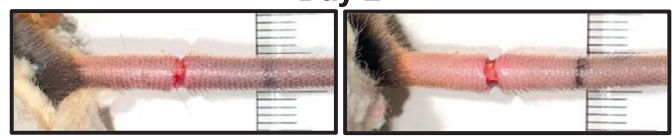

Day 10

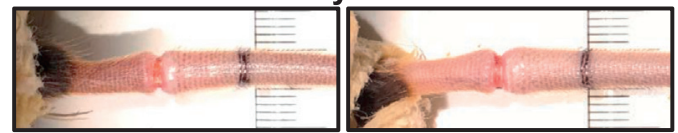

Day 20

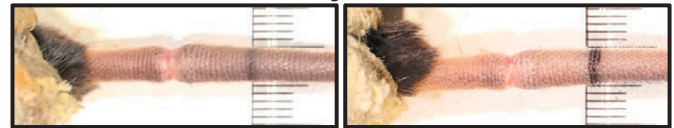

B

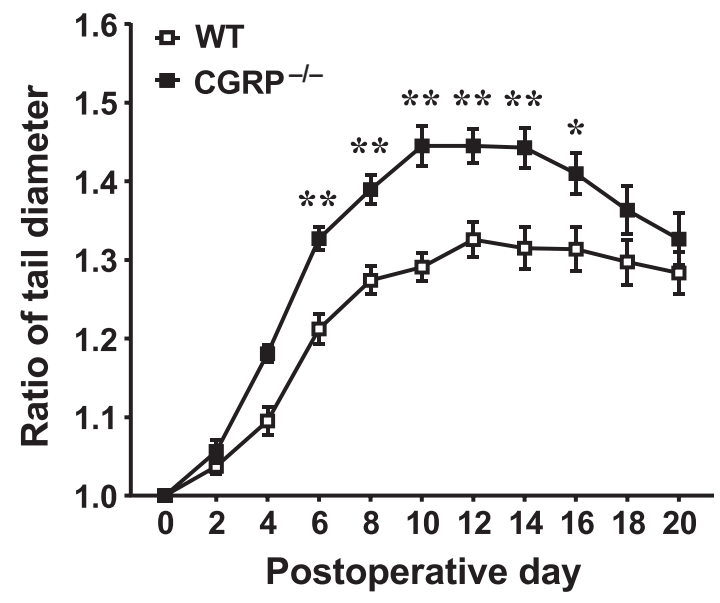

C Day 0

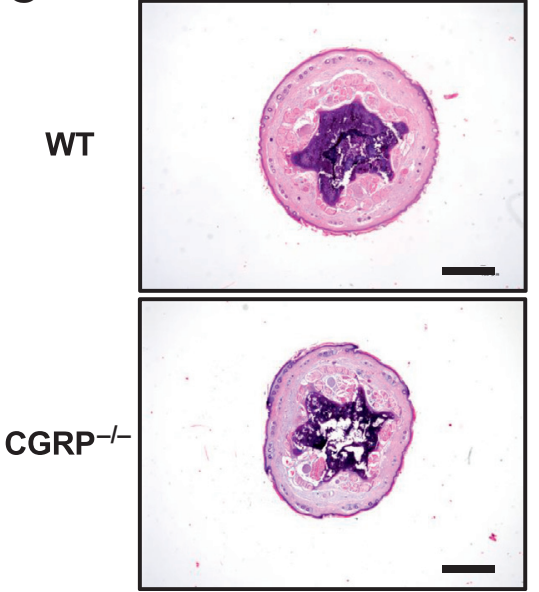

Day 10

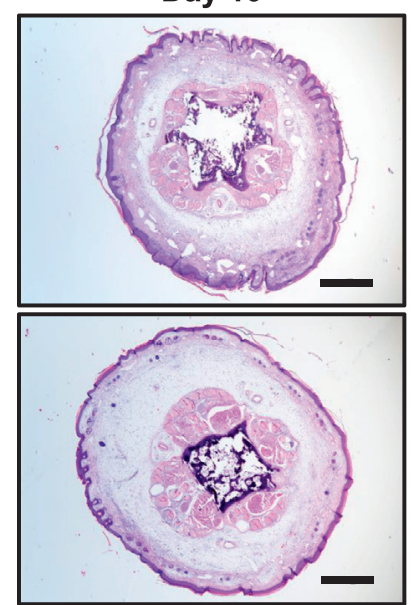

Day 20

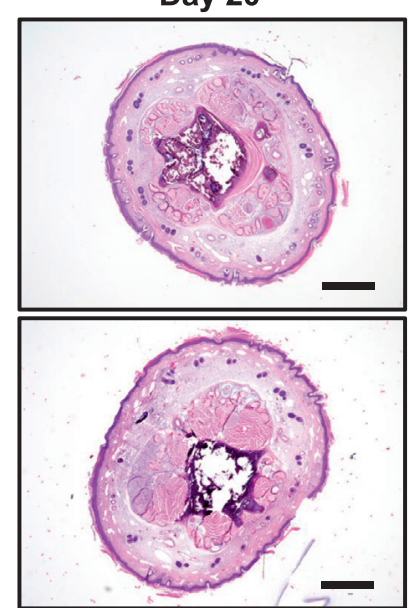

Figure 1 Severe postoperative tail lymphedema in calcitonin gene-related peptide knockout (CGRP-/-) mice. A: Appearance of the tails of wild-type (WT) and $\mathrm{CGRP}^{-1-}$ mice in the postoperative tail lymphedema model on day 0 (before surgery) and days 2, 10, and 20 (after surgery). B: Time course of the tail lymphedema. Shown are ratios of tail diameters on the indicated postoperative days to the diameter before surgery. Diameters were measured $1 \mathrm{~cm}$ distal to the wounds. C: Hematoxylin/eosin-stained cross sections from the tails of WT and CGRP ${ }^{-1-}$ mice in the postoperative tail lymphedema model on day 0 (before surgery) and days 10 and 20 (after surgery). Sections were collected 0.5 to $1 \mathrm{~cm}$ distal to the wounds. Data are expressed as means \pm SEM. $n=10$ in each group. ${ }^{*} P<0.01,{ }^{*} P<0.05$ (analysis of variance, followed by Sidak's test). Scale bars: $1 \mathrm{~mm}$ (C). D, distal side; $P$, proximal side.

Originally identified as a strong vasodilatory neuropeptide, CGRP is released primarily from sensory nerves and is widely distributed in the central and peripheral nervous systems. $^{3-5}$ In addition, CGRP is also expressed in the heart, lungs, digestive system, kidney, and blood vessels. ${ }^{6-8}$ Of the two major forms of CGRP, $\alpha$ and $\beta, \alpha$-CGRP is predominantly expressed throughout the central and peripheral nervous systems, whereas $\beta$-CGRP is found mainly in the enteric nervous system. ${ }^{9,10}$ CGRP exhibits a wide variety of biological effects in addition to its vasodilatory activity. For example, it now appears to be involved in the pathophysiology of migraine, ${ }^{11}$ and in the heart it may play a protective role against myocardial ischemia and heart failure. ${ }^{12,13}$ CGRP also reportedly facilitates angiogenesis in response to ischemia, ${ }^{14,15}$ improves blood flow and vascular endothelial function, ${ }^{16,17}$ and plays an important role in inflammation. ${ }^{18,19}$

From its structural homology and similar vasodilatory effects, CGRP is thought to be part of the peptide family that also includes adrenomedullin (ADM). Like CGRP, $\mathrm{ADM}$ is secreted by numerous tissues and organs and exerts a variety of effects. Moreover, CGRP, ADM, and other peptides in this family share a common seven transmembrane $\mathrm{G}$ protein-coupled receptor, calcitonin receptor-like receptor (CLR). ${ }^{20}$ The specific affinities of CLR for its various ligands are determined by three accessory proteins called receptor activity-modifying proteins (RAMP1-3). ${ }^{20}$ When associated with RAMP1, 
A

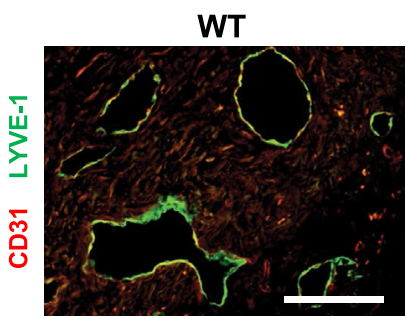

CGRP $^{-1-}$

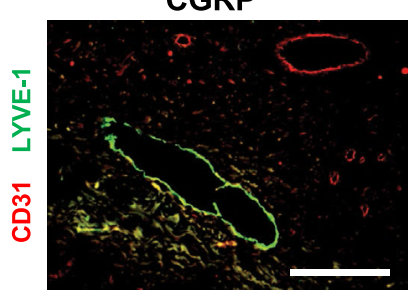

D

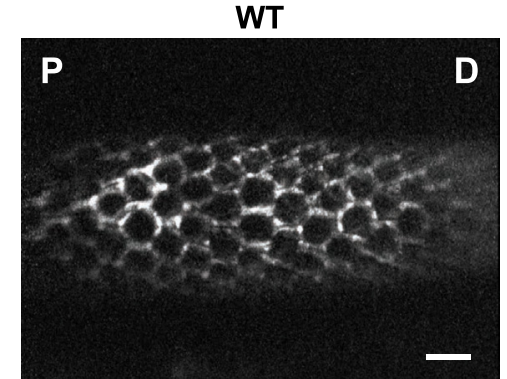

D
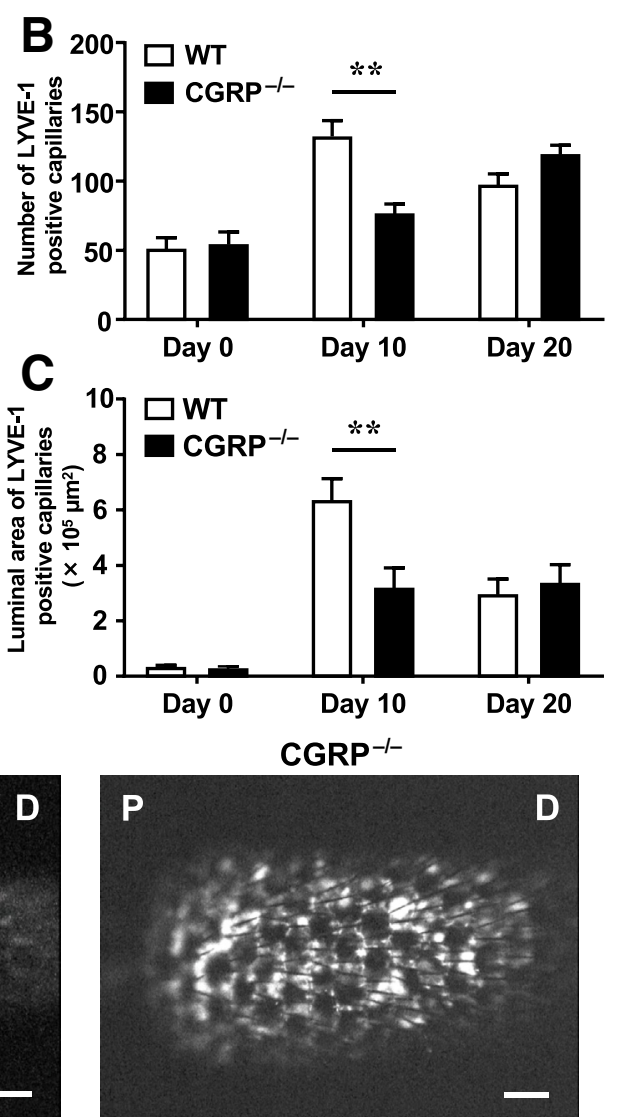

Figure 2 Fewer lymphatic capillaries in postoperative lymphedematous tails of calcitonin gene-related peptide knockout $\left(\mathrm{CGRP}^{-/-}\right)$than wild-type (WT) mice. A: Fluorescent immunostained cross sections from the tails of WT and $\mathrm{CGRP}^{-/-}$mice on day 10 after surgery: red indicates CD31 (endothelial cell marker); green, lymphatic vessel endothelial hyaluronan receptor 1 (LYVE-1) (lymphatic endothelial cell marker). Sections were collected 0.5 to $1 \mathrm{~cm}$ distal to the wounds. B and C: Numbers (B) and (C) summations of luminal areas of LYVE-1-positive capillaries in a single cross section from the tails of WT and $\mathrm{CGRP}^{-1-}$ mice on days 0,10 , and 20. D: Indocyanine green (ICG) fluorescence micrographs of the tails of WT and CGRP ${ }^{-/-}$mice in the postoperative tail lymphedema model on day 10 after surgery. ICG was subcutaneously injected into the tail tip. Lymphatic imaging of the tail over an area extending from 1 to $2 \mathrm{~cm}$ distal to the wound was observed 30 minutes after the injection. Data are expressed as means \pm SEM. $n=5$ in each group (B and $\mathbf{C}$ ). ${ }^{* *} P<0.01$ (analysis of variance, followed by Sidak's test). Scale bars: $100 \mu \mathrm{m}$ (A); 1 mm (D). D, distal side; P, proximal side.
CLR has higher affinity for CGRP; association with RAMP2 or RAMP3 gives CLR a higher affinity for ADM.

With the use of RAMP1 knockout (-/-) mice, Kurashige et $\mathrm{al}^{21}$ provided evidence that RAMP1 expressed by macrophages is an important mediator of lymphatic capillary formation during skin wound healing. However, the RAMP2/CLR and RAMP3/CLR complexes also have affinity for CGRP, although lower than that of RAMP1/CLR complex. In addition, the RAMP1/CLR complex also functions as a receptor for other members of the calcitonin superfamily, including calcitonin, intermedin, and amylin. Consequently, it remains unclear whether CGRP directly regulates lymphatic capillary formation. Clarification of the precise function of CGRP in lymphatic capillary formation is necessary for the potential therapeutic application of CGRP to the treatment of lymphedema. In this study, therefore, $\mathrm{CGRP}^{-/-}$mice were used to analyze the actions of endogenous CGRP in postoperative lymphedema.

\section{Materials and Methods}

\section{Experimental Animals}

CGRP is encoded by the same gene as calcitonin. $\alpha-\mathrm{CGRP}^{-1-}$ mice were generated with the use of a targeting
DNA construct that replaced exon 5, encoding a CGRPspecific region, to prevent the effects of calcitonin deficiency. ${ }^{22}$ C57BL/6J pure-background male mice were used.

Male 8- to 10-week-old $\mathrm{CGRP}^{-1-}$ mice and their wildtype (WT) littermates were used in this study. All animal experiments were performed in accordance with the ethical guidelines of Shinshu University. Before all invasive procedures, mice were anesthetized through intraperitoneal injection of 2,2,2-tribromoethanol (240 mg/kg; Wako, Osaka, Japan).

\section{Postoperative Tail Lymphedema Model}

Tail lymphedema was induced as described previously with modification. ${ }^{23-25}$ In brief, a 3-mm-wide ring of skin was removed $1 \mathrm{~cm}$ distal to the base of the tail. Mouse tail skin consists of a regular and hexagonal network of dermal lymphatic capillaries. ${ }^{26-28}$ A circumferential incision was made through the dermis to sever the dermal lymphatic capillaries without damaging the major blood or lymphatic vessels, which are located deeper within the tail, close to the bony/cartilaginous portion. Care was taken to maintain the integrity of the major underlying vessels and tendons so that the tail distal to the incision did not become necrotic. The injured region of the tail was then wrapped with adhesive tape to protect the surgical site from infection. The horizontal tail diameter was measured with a Vernier 


\section{Calca (CGRP)}

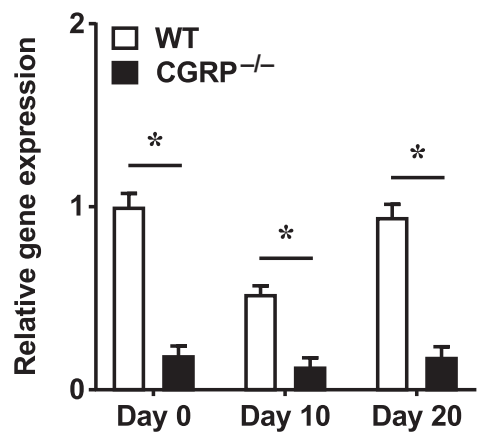

Ramp1

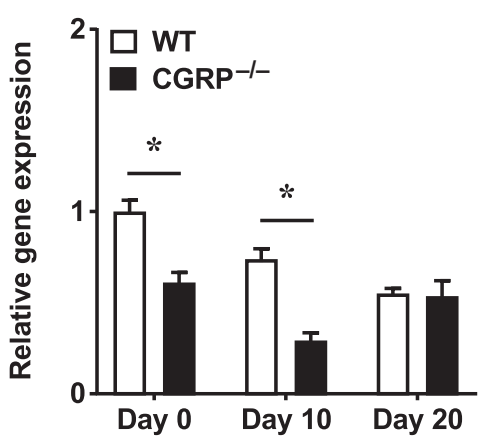

Adm (Adrenomedullin)

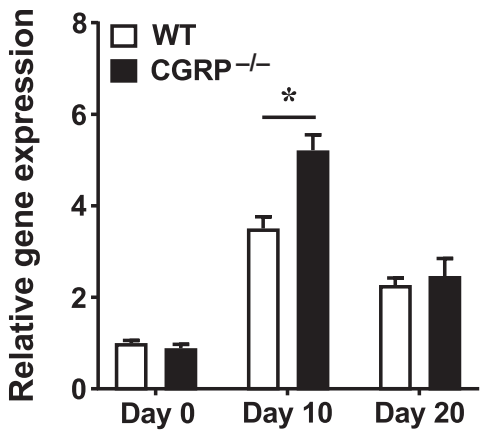

Ramp2

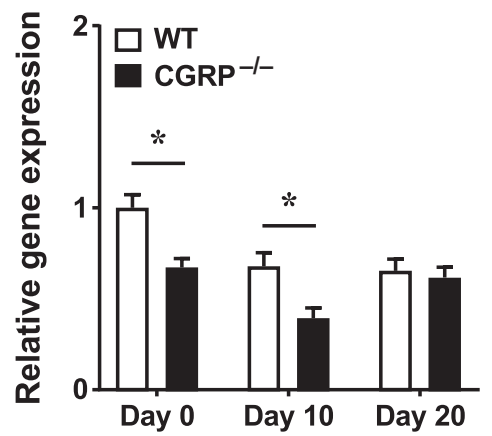

Calcr (CLR)

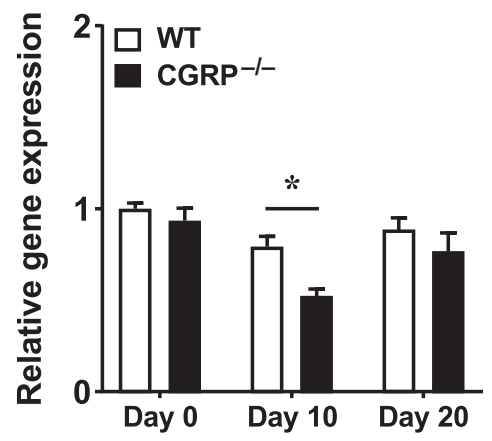

\section{Ramp3}

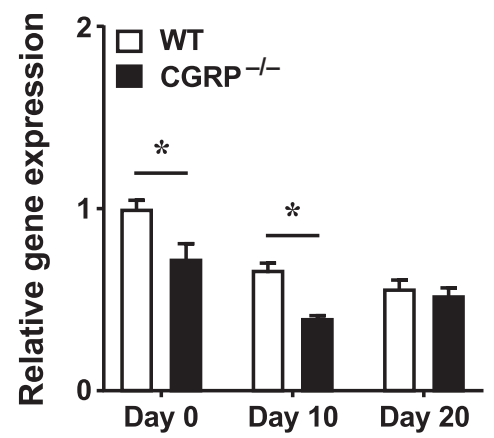

Figure 3 Expression of calcitonin gene-related peptide (CGRP) and related genes in postoperative lymphedematous tails. Expression levels of mRNAs encoding CGRP, ADM, CLR, receptor activity-modifying protein (RAMP)1, RAMP2, and RAMP3 in the tails of wild-type (WT) and CGRP ${ }^{-/-}$mice on day 0 (before surgery) and days 10 and 20 after surgery. Tissues were collected from the tails in a region from 1 to $2 \mathrm{~cm}$ distal to the wounds, and mRNA levels were determined with real-time RT-PCR. Data from the WT mice on day 0 were assigned a value of 1 . Data are expressed as means \pm SEM. $n=9$ for Adm (Adrenomedullin); $n=8$ for Calca (CGRP); and $n=10$ for Calcr (CLR), Ramp1, Ramp2, and Ramp3. ${ }^{*} P<0.05$ (analysis of variance, followed by Sidak's test).

caliper $1 \mathrm{~cm}$ from the distal edge of the injury, where a circumferential mark was made on the tail. For pathologic examination, sections of the complete tail were collected 0.5 to $1 \mathrm{~cm}$ distal to the wounds. For gene expression analysis, tail skin and subcutaneous tissues (without muscle, bone, or cartilage) were collected 1 to $2 \mathrm{~cm}$ distal to the wounds.

\section{Selective Depletion of M2 Macrophages}

Mannosylated clodronate liposomes (MCLs) selectively deplete M2 macrophages. ${ }^{29}$ MCLs or control liposomes (Encapsular Nano Sciences, Brentwood, TN) were injected into the tail vein. In each mouse, $0.01 \mathrm{mg} / \mathrm{g}$ MCLs were injected 3 days before (day -3 ) and on days 0,4 , and 7 after the operation.

\section{Histologic Analysis}

Tissues were fixed overnight in $4 \%$ paraformaldehyde, embedded in paraffin, and cut into 5 - $\mu \mathrm{m}-$ thick sections for histologic examination. The specimens were then deparaffinized for hematoxylin/eosin staining or immunohistochemistry with anti-CD31 (BD Biosciences, San Jose, CA), anti-LYVE-1 (Relia Tech GmbH, Wolfenbüttel Germany), anti- $\alpha$-CGRP (Sigma-Aldrich, St. Louis, MO), anti-CD68 antibody (Abcam, Cambridge, UK), anti-F4/80 (Bio-Rad, Hercules, CA), anti-CD11c (Abcam), anti-Arginase1 (Genetex, Irvine, CA), or anti-CD206 antibodies (Abcam). Nuclei were stained with DAPI (Life Technologies, Carlsbad, CA). Fluorescence was observed with a KEYENCE model BZ-X710 fluorescence microscope (Osaka, Japan) equipped with the appropriate filter sets. Measurements of the luminal areas of capillaries and numbers of macrophages were made with Image $\mathbf{J}$ software version 1.52 (https://imagej.nih.gov/ij/download.html).

Visualization of the Lymphatic Capillaries Using Intradermally Injected Indocyanine Green

To examine the morphologic structure of tail lymphatic capillaries, the tails of anesthetized mice were injected intradermally with $10 \mu \mathrm{L}$ of indocyanine green (DaiichiSankyo, Tokyo, Japan) at a site $3 \mathrm{~cm}$ from the base of the tail. Images were then taken with a Heidelberg Retina 

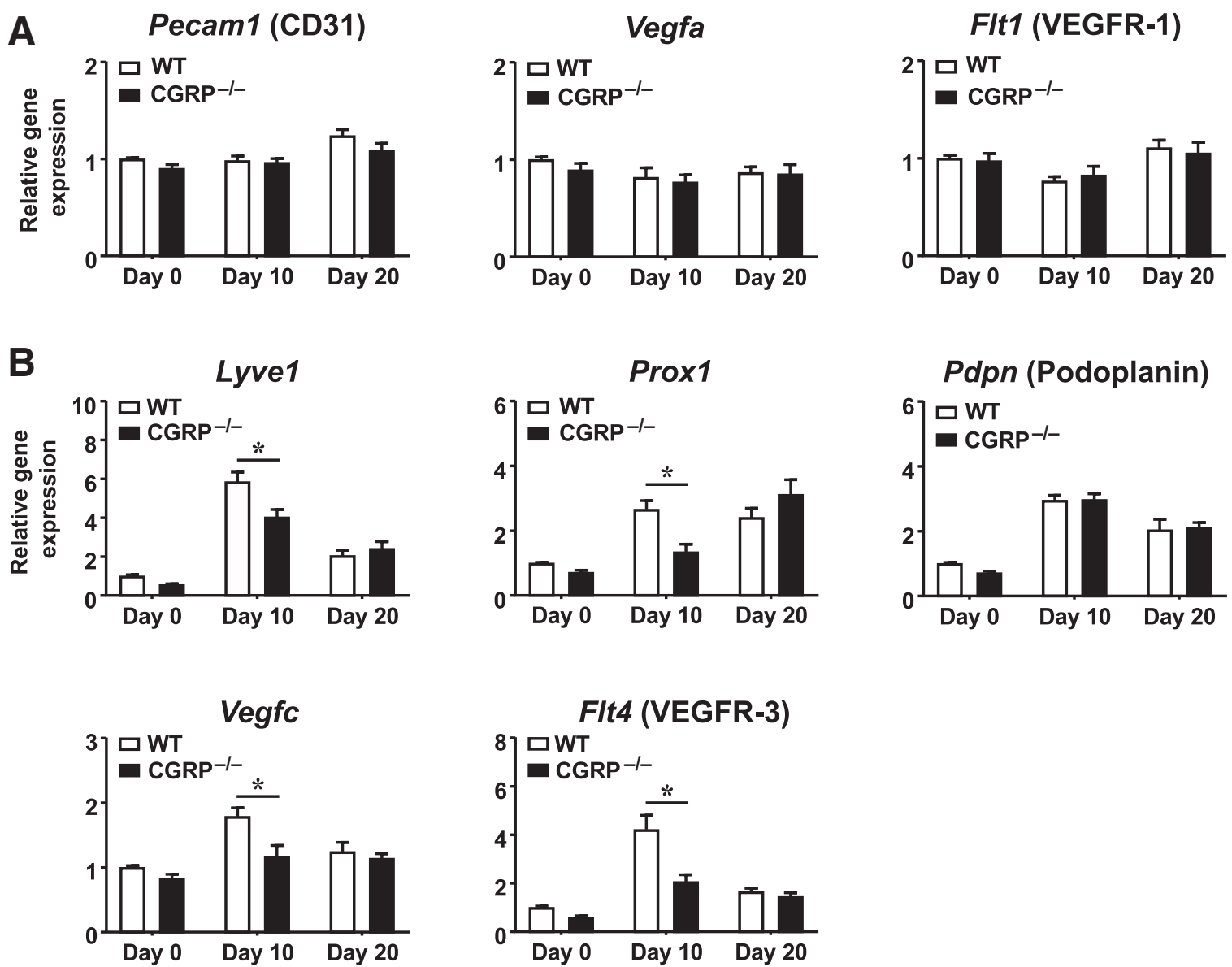

Figure 4 Expression of angiogenesis-related and lymphatic capillary formation-related genes in postoperative lymphedematous tails. A: Expression levels of mRNAs encoding CD31, vascular endothelial growth factor (VEGF)-A, and vascular endothelial growth factor receptor (VEGFR)-1 in the postoperative lymphedematous tails of wild-type (WT) and calcitonin gene-related peptide knockout (CGRP ${ }^{-}$) mice on day 0 (before surgery) and days 10 and 20 after surgery. Tissues were collected from a region 1 to $2 \mathrm{~cm}$ distal to the wounds, and mRNA levels were determined with real-time RT-PCR. Data from the WT mice on day 0 were assigned a value of 1. B: Expression levels of mRNAs encoding lymphatic vessel endothelial hyaluronan receptor 1 (LYVE-1), PROX1, Podoplanin, VEGF-C, and VEGFR-3 in the postoperative lymphedematous tails of WT and CGRP ${ }^{-1-}$ mice on day 0 (before surgery) and days 10 and 20 after surgery. Tissues were collected from a region 1 to $2 \mathrm{~cm}$ distal to the wounds, and mRNA levels were determined with real-time RT-PCR. Data from the WT mice on day 0 were assigned a value of 1. Data are expressed as means \pm SEM. $n=9$ for Pecam1 (CD31) (A); $n=10$ for Vegfa and Flt1 (VEGFR-1) (A); $n=10$ for Lyve1, Pdpn (Podoplanin), Vegfc, and Flt4 (VEGFR-3) (B); $n=9$ for Prox1 (B). ${ }^{*} P<0.05$ (analysis of variance, followed by Sidak's test).

Angiograph 2 (Heidelberg Engineering GmbH, Heidelberg, Germany) 30 minutes after the tail injection.

\section{RNA Extraction and Real-Time Quantitative RT-PCR Analysis}

Total RNA was extracted from tissues with the use of Trireagent (Molecular Research Center, Inc., Cincinnati, $\mathrm{OH})$, after which time the RNA was treated with DNA-Free (Ambion, Naugatuck, CT) to remove contaminating DNA and was reverse transcribed with a High Capacity cDNA Reverse Transcription Kit (Applied Biosystems, Foster City, CA). Real-time quantitative RT-PCR was performed with an Applied Biosystems 7300 real-time PCR System with SYBR green (Toyobo, Osaka, Japan) or Realtime PCR Master Mix (Toyobo) and TaqMan probes (MBL, Aichi, Japan). The primers and probes used are listed in Table 1. Values were normalized to mouse glyceraldehyde-3- phosphate dehydrogenase (Pre-Developed TaqMan assay reagents; Applied Biosystems).

\section{Statistical Analysis}

Statistical analysis was performed with GraphPad Prism software version 7.03. (GraphPad Software Inc., San Diego, CA). Values are expressed as means \pm SEM. Comparisons among multiple groups were made with analysis of variance, followed by Sidak's test. Comparisons between two groups were made with $t$-test. $P<0.05$ was considered statistically significant.

\section{Results}

Severe Postoperative Tail Lymphedema with Reduced Lymphatic Capillary Formation in $\mathrm{CGRP}^{-/-}$Mice

The tails of mice contain a highly regular network of lymphatic capillaries in the skin. Consequently, 
A

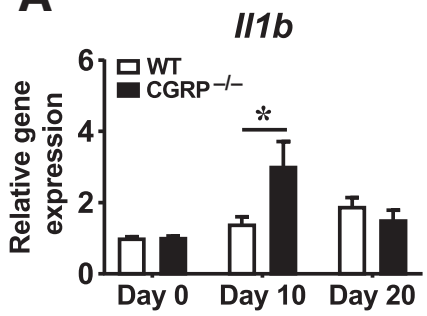

116

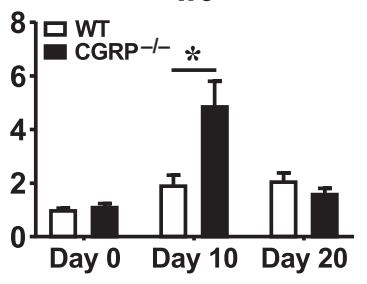

Tnf

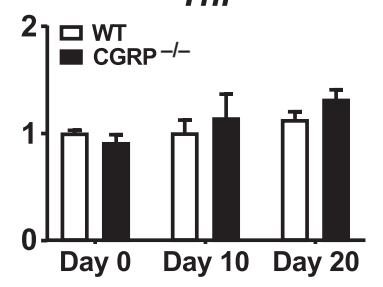

II10

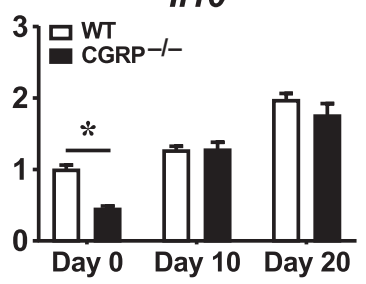

B
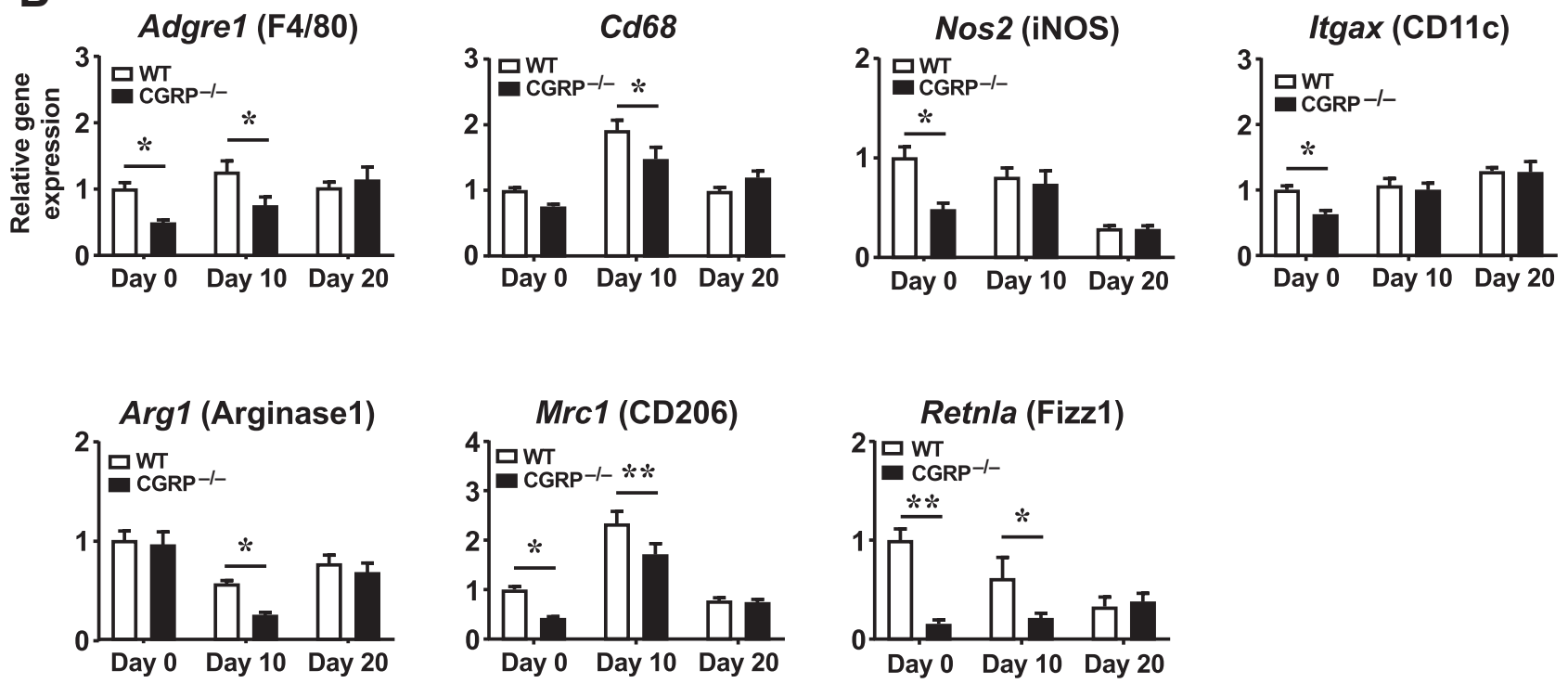

Figure 5 Expression of cytokines and macrophage markers in postoperative lymphedematous tails. A: Expression levels of mRNAs encoding IL-1 $\beta$, IL-6, tumor necrosis factor (TNF) $\alpha$, and IL-10 in the postoperative lymphedematous tails of wild-type (WT) and calcitonin gene-related peptide knockout $\left(\mathrm{CGRP}^{-1-}\right)$ mice on day 0 (before surgery) and days 10 and 20 after surgery. Tissues were collected from a region 1 to $2 \mathrm{~cm}$ distal to the wounds, and mRNA levels were determined with real-time RT-PCR. Data from the WT mice on day 0 were assigned a value of 1. B: Expression levels of mRNAs encoding F4/80 and CD68 (pan-macrophage markers), CD11C and inducible nitric oxide synthase (iNOS) (M1 macrophage markers), and CD206, Arginase1, and Fizz1 (M2 macrophage markers) in the postoperative lymphedematous tails of WT and CGRP ${ }^{-1-}$ mice on day 0 (before surgery) and days 10 and 20 after surgery. Tissues were collected in a region 1 to $2 \mathrm{~cm}$ distal to the wounds, and mRNA levels were determined with real-time RT-PCR. Data from the WT mice on day 0 were assigned a value of 1. Data are expressed as mean \pm SEM. $n=8$ for Il1b, Il6, Tnf, and Il10 (A); $n=9$ for Adgre1 (F4/80) and Nos2 (iNOS) (B); $n=8$ for Arg1 (Arginase1) (B); $n=7$ for Cd68, Itgax (CD11C), Mrc1 (CD206), and Retnla (Fizz1) (B). ${ }^{*} P<0.05, * * P<0.01$ (analysis of variance, followed by Sidak's test).

circumferential removal of the skin from the tail obstructs lymphatic flow, resulting in acute lymphedema and enabling this system to serve as a model of secondary lymphedema. ${ }^{23}$ The time course of postoperative lymphedema was evaluated by measuring tail diameters in $\mathrm{WT}$ and $\mathrm{CGRP}^{-1-}$ mice. After the surgical procedure, the tails of all treated mice became edematous, with the edema reaching a peak on postoperative days 10 to 12 in both WT and $\mathrm{CGRP}^{-1-}$ mice. Thereafter, the tail edema gradually resolved. Notably, from postoperative day 6 to day 16, tail thickness was significantly greater in $\mathrm{CGRP}^{-1-}$ mice than in WT mice (Figure 1, A and B). Cross sections of the tails also revealed enhanced postoperative edema in $\mathrm{CGRP}^{-l-}$ mice (Figure 1C). Although no difference between WT and $\mathrm{CGRP}^{-1-}$ mice was detected before surgery (day 0 ), the tails of $\mathrm{CGRP}^{-1-}$ mice showed significantly greater swelling on postoperative day 10 .

With the use of this tail lymphedema model, the lymphatic capillaries within lymphedematous sites were visualized by immunostaining sections for the lymphatic marker LYVE-1 (Figure 2A). Although the numbers of LYVE-1-positive lymphatic capillaries (Figure 2B) and the sums of their luminal areas (Figure 2C) were increased after the surgery in both WT and $\mathrm{CGRP}^{-1-}$ mice, they were significantly smaller in $\mathrm{CGRP}^{-/-}$mice than in WT mice on day 10. Immunostaining was also performed for CD31 to assess changes in blood vessels within the injury site, but neither the numbers nor the luminal areas differed between WT and CGRP $^{-l-}$ mice (Supplemental Figure S1, A and B). These results clearly indicated specific impairment of 
A
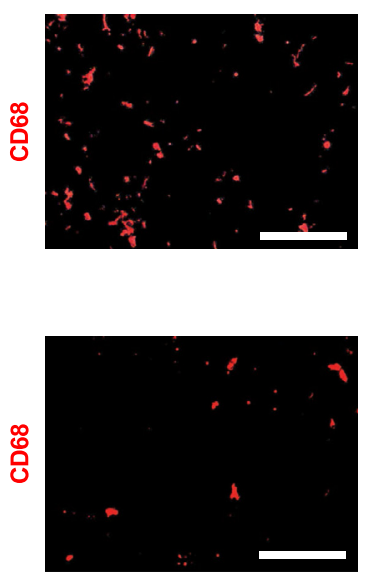

WT

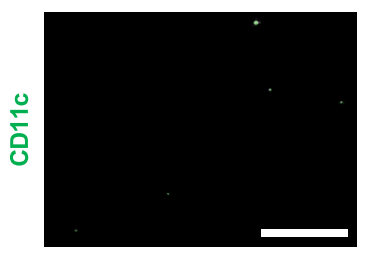

CGRP $^{-1-}$

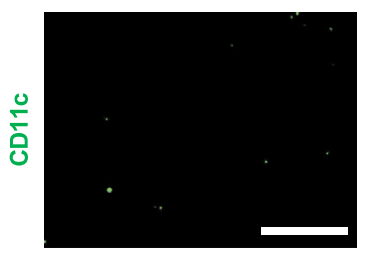

B

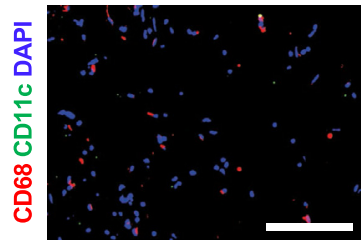

C

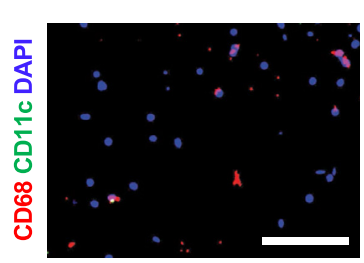

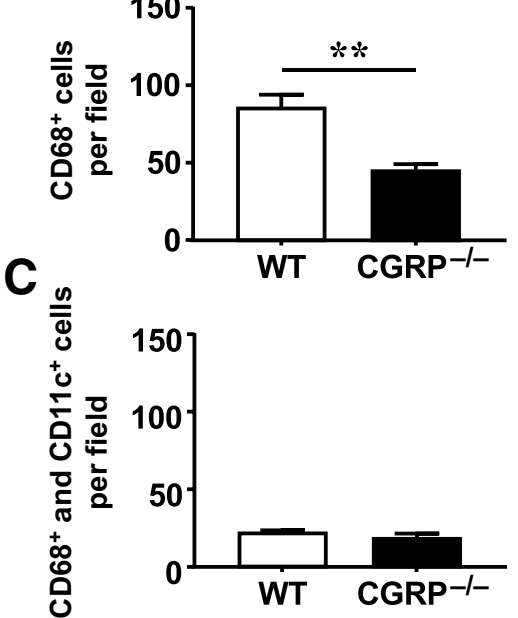

Figure 6 M1 macrophage numbers are unchanged in calcitonin gene-related peptide knockout (CGRP $\left.{ }^{-/}\right)$mice. A: Fluorescently immunostained cross sections from the tails of wild-type (WT) and $\mathrm{CGRP}^{-1-}$ mice in the postoperative lymphedema model on day 10 after surgery: red indicates CD68 (panmacrophage marker); green, CD11c (M1 macrophage marker); blue, DAPI. Sections were collected 0.5 to $1 \mathrm{~cm}$ distal to the wounds. B: Numbers of CD68 ${ }^{+}$cells in cross sections from the tails of WT and CGRP ${ }^{-1-}$ mice on postoperative day 10. C: Numbers of $\mathrm{CD}^{+} 8^{+}$and CD11c ${ }^{+}$cells in cross sections from the tails of WT and GGRP $^{-1-}$ mice on day 10 after surgery. Data are expressed as means \pm SEM. $n=5$ in each group (B and $\left.\mathbf{C}\right) .{ }^{* *} P<0.01$ ( $t$-test). Scale bars: $100 \mu \mathrm{m}(\mathbf{A})$.

lymphatic capillary formation with no effect on angiogenesis in CGRP ${ }^{-1-}$ mice.

To analyze the morphologic structure of lymphatic capillaries, indocyanine green was subcutaneously injected into the tail tip and then observed with fluorescence microscopy 30 minutes after the injection. WT mice showed a honeycomb-like pattern of lymphatic vessels, whereas this pattern was disrupted in $\mathrm{CGRP}^{-1-}$ mice (Figure 2D).

Altered Expression of CGRP and its Related Factors in the Postoperative Lymphedematous Tails of $\mathrm{CGRP}^{-/-}$Mice

The gene expression levels of Calca (CGRP), Adm (Adrenomedullin), Calcr (CLR), Ramp1, Ramp2, and Ramp3 were analyzed in the lymphedematous tails of WT and $\mathrm{CGRP}^{-l-}$ mice (Figure 3). Calca (CGRP) expression was significantly lower in $\mathrm{CGRP}^{-1-}$ than in WT mice, although a slight and nonspecific signal was detected, which is consistent with earlier observations. ${ }^{30}$ Expression of Adm (Adrenomedullin), whose functions somewhat overlap those of CGRP, was significantly elevated in $\mathrm{CGRP}^{-/-}$mice on postoperative day 10 , possibly as a compensatory response to the CGRP deficiency. However, components of the CGRP and ADM receptors [Calcr (CLR) and Ramp1-3] were all down-regulated in $\mathrm{CGRP}^{-1-}$ mice compared with WT mice. Immunostaining was also performed for CGRP to compare its distribution in WT and $\mathrm{CGRP}^{-1-}$ mice (Supplemental Figure S2, A and B). CGRP was significantly but transiently up-regulated in the postoperative lymphedematous tails of WT mice, with levels peaking on postoperative day 10. By contrast, no CGRP was detected in $\mathrm{CGRP}^{-1-}$ mice.

\section{Lymphatic Capillary Formation-Related Factors}

Are Down-Regulated in the Postoperative Lymphedematous Tails of $\mathrm{CGRP}^{-/-}$Mice

With the use of real-time RT-PCR, it was observed that in both WT and $\mathrm{CGRP}^{-1-}$ mice, expression of the angiogenesis-related factors Pecaml (CD31), Vegfa, and Flt1 [vascular endothelial growth factor receptor (VEGFR)1] was unaffected by the operative procedure (Figure 4A). By contrast, the lymphatic capillary formation-related factors Lyve1, Proxl, Vegfc, and Flt4 (VEGFR-3) were transiently up-regulated on postoperative day 10 . However, expression of these factors was significantly lower in $\mathrm{CGRP}^{-1-}$ than in WT mice on postoperative day 10 (Figure 4B).

\section{Altered Cytokine Expression in $\mathrm{CGRP}^{-/-}$Mice}

Analysis of cytokine expression revealed that, although the expression of the inflammatory cytokine $I l l b$ was slightly up-regulated on postoperative day 10 in WT mice, the upregulation was significantly more prominent in $\mathrm{CGRP}^{--}$ mice (Figure 5A). However, expression of antiinflammatory cytokine $\mathrm{IllO}$ was significantly lower in $\mathrm{CGRP}^{-1-}$ than in WT mice before surgery (day 0 ). These results suggested there was greater postoperative inflammation in $\mathrm{CGRP}^{-1-}$ mice than in WT mice.

\section{M2 Macrophages Are Reduced in the Postoperative Lymphedematous Tails of $\mathrm{CGRP}^{-/-}$Mice}

The expression of macrophage markers in the postoperative lymphedema model was also analyzed (Figure 5B). The 
A
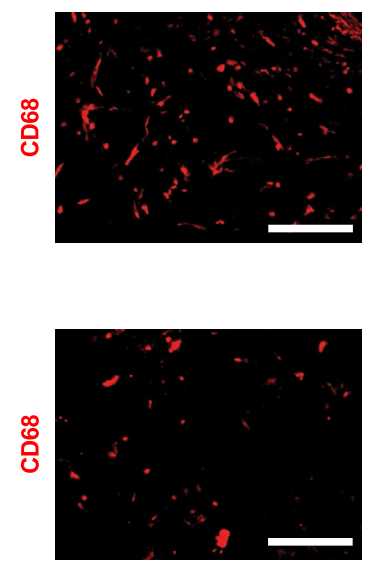

D
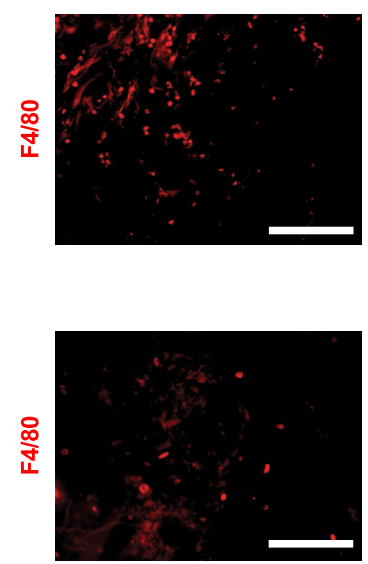

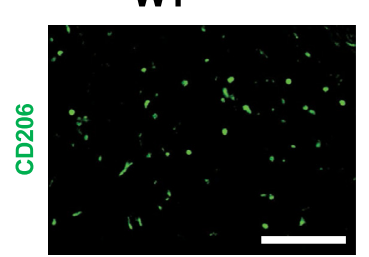

CGRP $^{-1-}$

WT

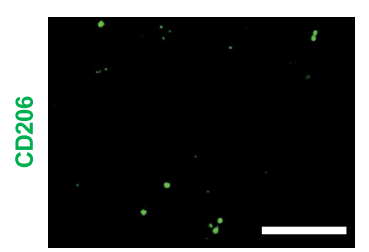

WT

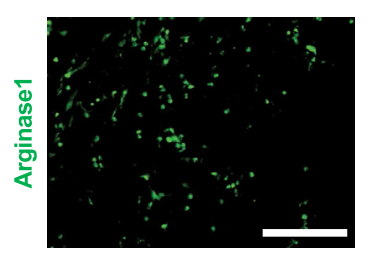

$\mathrm{CGRP}^{-1-}$

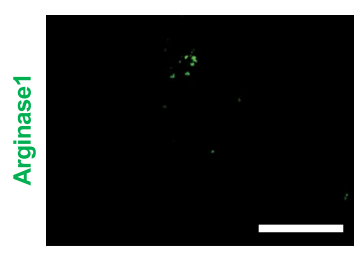

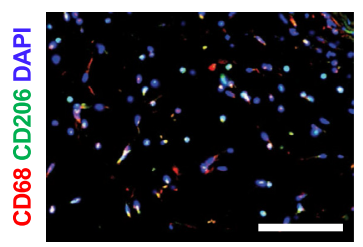
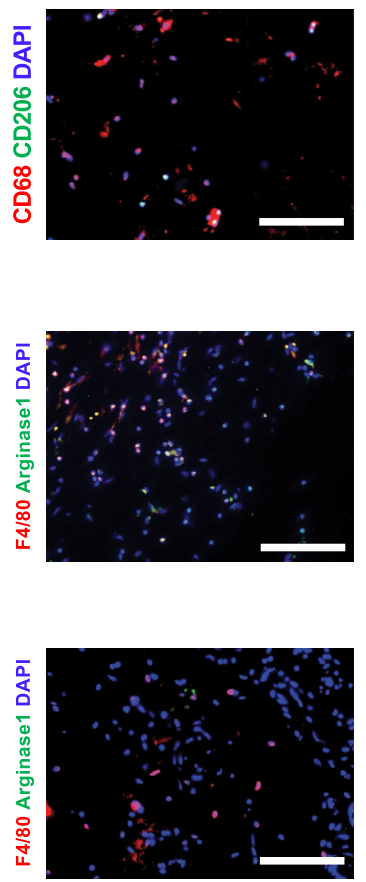

B

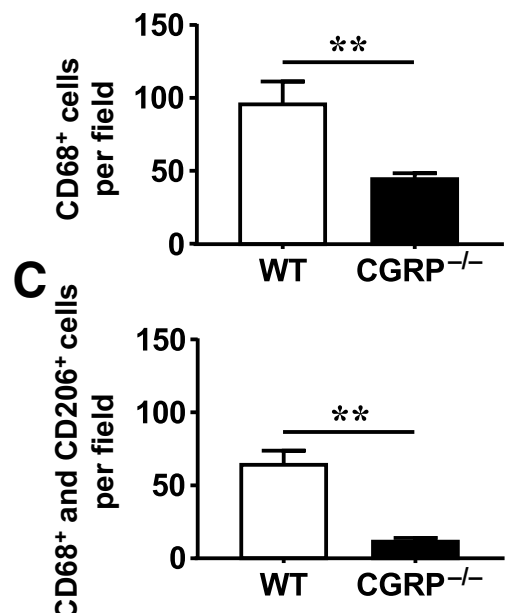

E

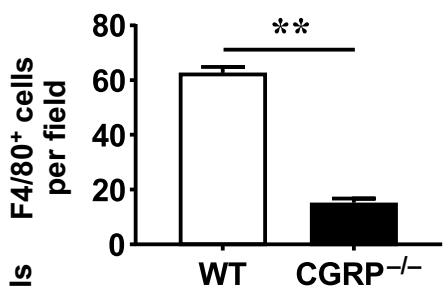

$F \stackrel{0}{\frac{0}{\Phi}}$

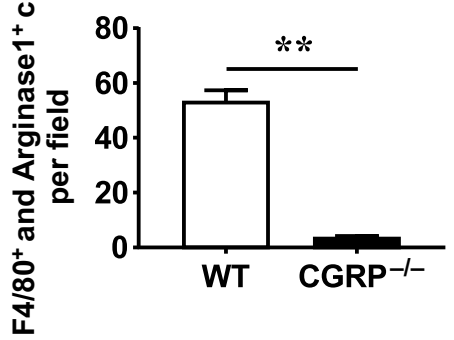

Figure 7 M2 macrophage numbers are decreased in calcitonin gene-related peptide knockout (CGRP $\left.{ }^{-/-}\right)$mice. A: Fluorescently immunostained cross sections from the tails of wild-type (WT) and $\mathrm{CGRP}^{-/-}$mice in the postoperative lymphedema model on day 10 after surgery: red indicates CD68 (panmacrophage marker); green, CD206 (M2 macrophage marker); blue, DAPI. Sections were collected 0.5 to $1 \mathrm{~cm}$ distal to the wounds. B: Numbers of CD68 ${ }^{+}$cells in cross sections from the tails of WT and CGRP ${ }^{-/-}$mice on day 10 after surgery. C: Numbers of $\mathrm{CD}^{-} 8^{+}$and $\mathrm{CD}^{206^{+}}$cells in cross sections from the tails of WT and CGRP ${ }^{-/}$mice on postoperative day 10. D: Fluorescently immunostained cross sections from the tails of WT and CGRP ${ }^{-/-}$mice in the postoperative lymphedema model on day 10 after surgery: red indicates F4/80 (pan-macrophage marker); green, Arginase1 (M2 macrophage marker); blue, DAPI. Sections were collected 0.5 to $1 \mathrm{~cm}$ distal to the wounds. E: Numbers of F4/80-positive cells in cross sections from the tails of WT and CGRP ${ }^{-/-}$mice on day 10 after surgery. F: Numbers of F4/80 and Arginase1-double-positive ( $F 4 / 80^{+}$and Arginase $\left.1^{+}\right)$cells in cross sections from the tails of WT and CGRP ${ }^{-/-}$mice on postoperative day 10. Data are expressed as means \pm SEM. $n=5$ in each group (B, C, E, and F). Scale bars: $100 \mu \mathrm{m}(\mathbf{A}$ and D).

pan-macrophage markers Adgrel (F4/80) and Cd68 were significantly lower in $\mathrm{CGRP}^{-1-}$ mice than in WT mice before surgery (day 0 ) and on postoperative day 10. Although expression of Itgax (CD11c) and Nos2 (inducible nitric oxide synthase), two M1 macrophage markers, were reduced before the surgery in $\mathrm{CGRP}^{-/-}$mice, the difference became undetectable after the surgery. By contrast, expression of three M2 macrophage markers, Mrcl (CD206), Argl (Arginase1), and Retnla (Fizz1), were significantly lower in $\mathrm{CGRP}^{-1-}$ mice than in WT mice on postoperative day 10 . These observations suggested that the reduction in $\mathrm{F} 4 / 80$ and $\mathrm{CD} 68^{+}$macrophages in $\mathrm{CGRP}^{-/-}$ mice on postoperative day 10 mainly reflected a reduction of
M2 macrophages. To confirm this finding, double immunostaining was performed for the pan-macrophage marker CD68 and the M1 macrophage marker CD11c. The number of M1 macrophages positive for both CD68 and CD11c did not differ between WT and $\mathrm{CGRP}^{-1-}$ mice (Figure 6). Double-immunostaining was performed for the panmacrophage marker CD68 and the M2 macrophage marker CD206, or the pan-macrophage marker F4/80 and the M2 macrophage marker Arginase1. In contrast to M1 macrophages, numbers of M2 macrophages, positive for both CD68 and CD206 (Figure 7, A-C) or F4/80 and Arginase1 (Figure 7, D-F), were significantly lower in $\mathrm{CGRP}^{-1-}$ mice than in WT mice. 
A

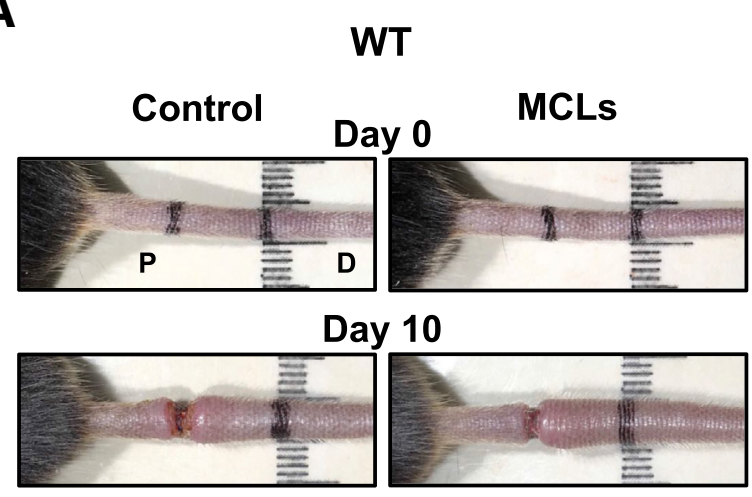

C

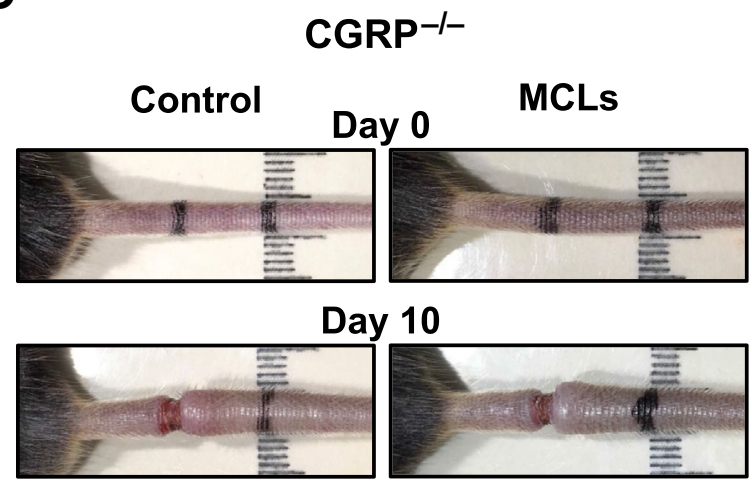

B

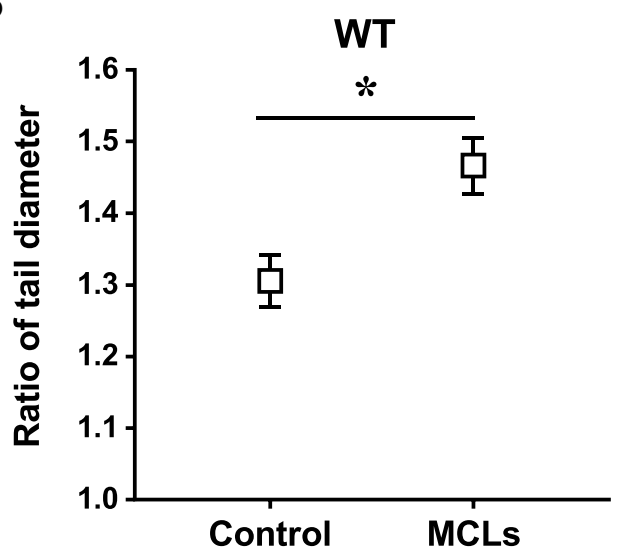

D

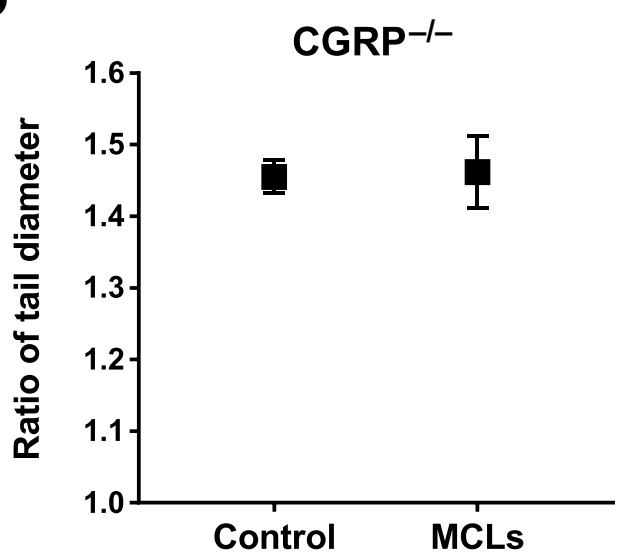

Figure 8 Administration of mannosylated clodronate liposomes (MCLs) exacerbates tail lymphedema in wild-type (WT) but not calcitonin gene-related peptide knockout (CGRP ${ }^{-1-}$ ) mice. A and C: Appearance of the tails of WT (A) and CGRP ${ }^{-1-}(\mathbf{C})$ mice in the postoperative tail lymphedema model on day 0 (before surgery) and day 10 (after surgery) with or without MCL administration. B and D: Ratios of tail diameters (day 10/day 0 ) in WT (B) and CGRP ${ }^{-/-}$(D) mice with or without MCLs. Diameters were measured $1 \mathrm{~cm}$ distal to the wounds. Data are expressed as means \pm SEM. $n=5$ in each group. ${ }^{*} P<0.05$ ( $t$-test). $D$, distal side; $P$, proximal side.

\section{M2 Macrophage Depletion Leads to Enhanced}

Postoperative Lymphedema in WT Mice but Not $\mathrm{CGRP}^{-/}$Mice

To clarify the relationship between the reduction in M2 macrophages and edema in $\mathrm{CGRP}^{-1-}$ mice, the effect of M2 macrophage depletion was assessed with MCLs in the postoperative lymphedema model. MCLs are selectively phagocytosed by M2 macrophages expressing mannose receptors, which causes cell death. ${ }^{29}$ MCL administration increased postoperative lymphedema in WT mice (Figure 8, A and B), but had little effect on the edema in $\mathrm{CGRP}^{-1}$ mice (Figure 8, $\mathrm{C}$ and $\mathrm{D}$ ). In cross sections of the tails, the numbers of LYVE1-positive lymphatic capillaries and the sums of their luminal areas were reduced by MCL administration in WT mice (Figure 9, A-C), whereas no changes were detected in CGRP $^{-l-}$ mice (Figure 9, D-F). Analysis of the macrophage distribution revealed that MCL administration had little effect on the number of $\mathrm{CD}_{6} 8^{+}$and $\mathrm{CD} 11 \mathrm{c}^{+} \mathrm{M} 1$ macrophages in either WT mice (Figure 10, $\mathrm{A}-\mathrm{C}$ ) or $\mathrm{CGRP}^{-1-}$ mice
(Figure 10, D-F). However, the numbers of $\mathrm{CD}^{+} 8^{+}$and $\mathrm{CD}^{206^{+}}$M2 macrophages were significantly reduced by MCL administration in WT mice (Figure 10, G-I), but not in $\mathrm{CGRP}^{-1-}$ mice (Figure 10, J-L).

Finally, the effect of MCLs was assessed on gene expression. MCL administration significantly reduced expression of the lymphatic capillary formation-related genes Lyve1, Pdpn (Podoplanin), Vegfc, and Flt4 (VEGFR-3) in WT mice (Figure 11A), but not in $\mathrm{CGRP}^{-1-}$ mice (Figure 11B). Expression of macrophage markers also revealed specific MCL-induced down-regulation of the M2 macrophage marker Argl (Arginase1) in WT mice (Figure 11C), whereas expression levels of both M1 and M2 macrophage markers were unchanged in $\mathrm{CGRP}^{-/-}$mice (Figure 11D).

\section{Discussion}

The lymphatic system is responsible for the maintenance of tissue fluid balance. When lymphatic capillaries are 
A

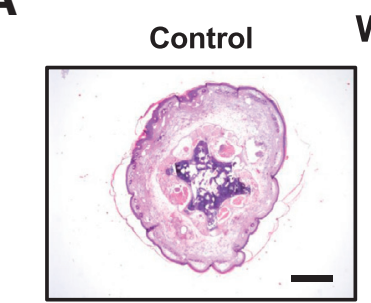

Control

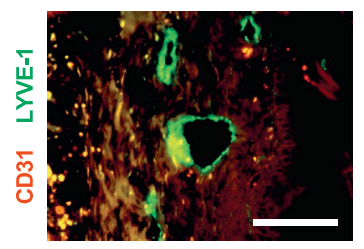

WT

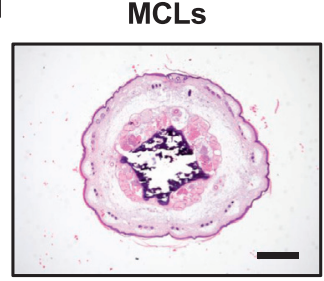

MCLs
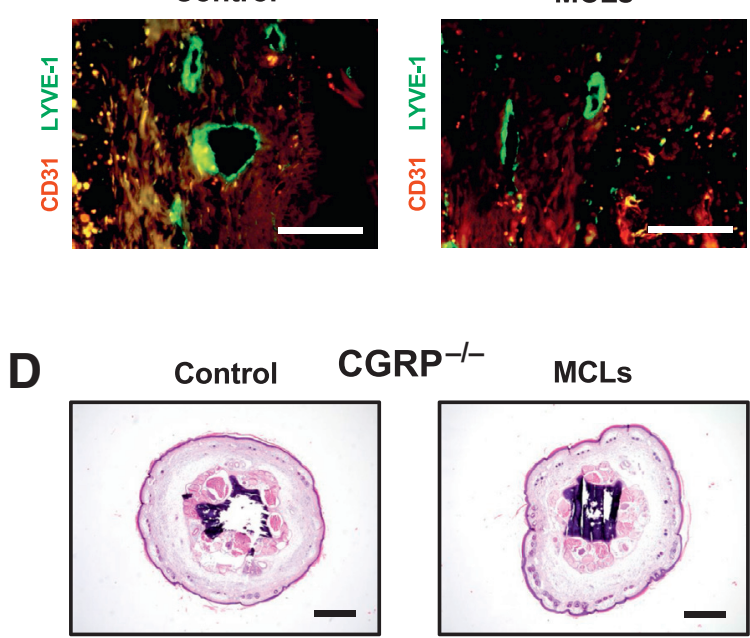

Control

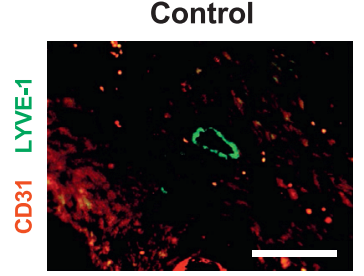

$\mathrm{CGRP}^{-1-} \quad$ MCLs

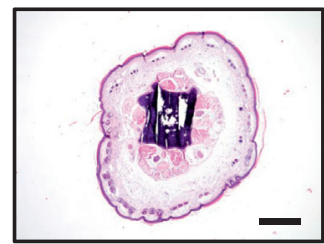

MCLs

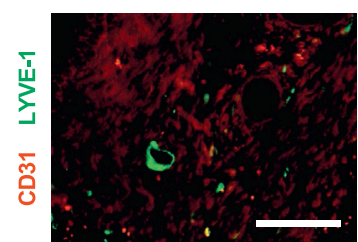

B

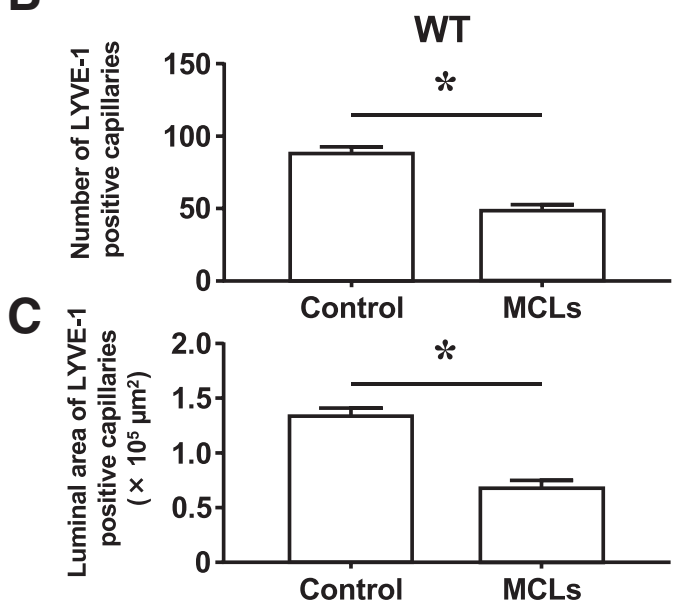

E

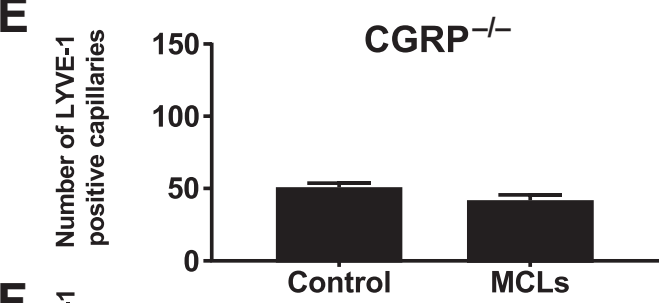

$\mathbf{F}$

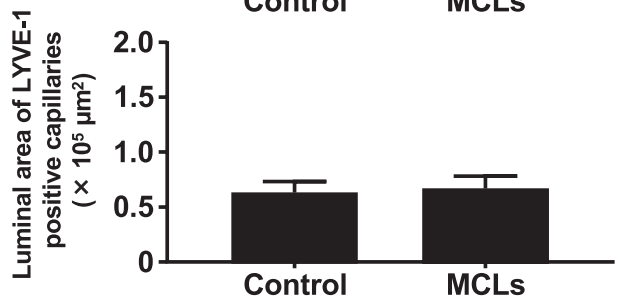

Figure 9 Mannosylated clodronate liposome (MCL) administration suppresses lymphatic capillary formation in wild-type (WT) but not calcitonin gene-related peptide knockout (CGRP ${ }^{-1-}$ ) mice. A and $\mathbf{D}$ : Hematoxylin/eosin-stained cross sections of the lymphedematous tails (upper panels) and immunofluorescence staining of CD31 and lymphatic vessel endothelial hyaluronan receptor 1 (LYVE-1; lower panels) from WT (A) and CGRP ${ }^{-/-}$(D) mice on postoperative day 10, with or without MCL administration: red indicates CD31; green, LYVE-1. Sections were collected at tails 0.5 to $1 \mathrm{~cm}$ distal to the wounds. B and C: Numbers (B) and summations of luminal areas (C) of LYVE-1-positive lymphatic capillaries in single cross sections of the lymphedematous tails of WT mice on postoperative day 10, with or without MCLs. $\mathbf{E}$ and F: Numbers (E) and summations of luminal areas (F) of LYVE-1-positive lymphatic capillaries in single cross sections of the lymphedematous tails of CGRP ${ }^{-/}$mice on postoperative day 10 , with or without MCLs. Data are expressed as means \pm SEM. $n=5$ in each group (B, C, E, and $\mathbf{F}$ ). ${ }^{*} P<0.05$ (t-test). Scale bars: $1 \mathrm{~mm}$ (A and $\mathbf{D}$, upper panels); $100 \mu \mathrm{m}$ (A and $\mathbf{D}$, lower panels).

damaged due to invasive surgeries or radiation therapy, severe fluid accumulation can be evoked in the affected tissues. This is known as secondary lymphedema and is characterized by gross swelling of the affected peripheral area, commonly the limbs. ${ }^{31}$ Lymphedema patients are susceptible to soft tissue infections, and many patients need constant prophylactic antibiotics. Chronic neuropathic pain is another common symptom of lymphedema. ${ }^{32}$ But despite its clinical importance, methods for treating lymphedema remain limited. An advanced surgical procedure, supermicrosurgical lymphaticovenular anastomosis, has been developed, ${ }^{1,2}$ but the operation is not curative, and there are few other therapeutic options. Consequently, establishment of novel treatment methods for secondary lymphedema is badly needed. This study focused on CGRP as a candidate to promote lymphatic capillary formation.

With the use of $\mathrm{CGRP}^{-1-}$ mice in the present study, the importance of endogenous CGRP for lymphatic capillary formation was revealed in a mouse model of postoperative lymphedema, which suggested the therapeutic potential of CGRP. Another member of the calcitonin superfamily, ADM, possesses both angiogenic and lymphangiogenic activities. $^{33-37}$ CGRP and ADM share a common receptor, CLR. The specific affinity of CLR for its ligands is determined by RAMP1-3. ${ }^{20}$ Association with RAMP2 or RAMP3 gives CLR a higher affinity for ADM. RAMP2 ${ }^{-1-}$ mice die in utero due to blood vessel abnormalities, which is indicative of its essential role in vascular development. ${ }^{33,38}$ 
A
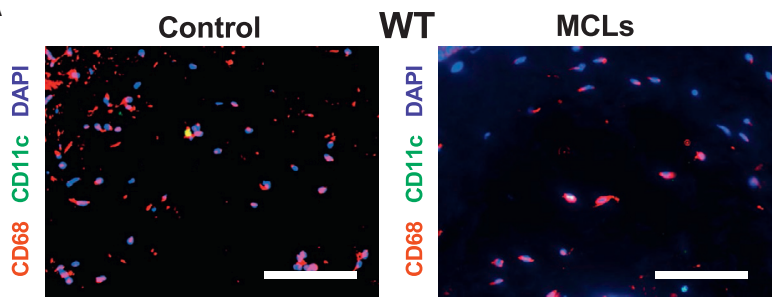

D
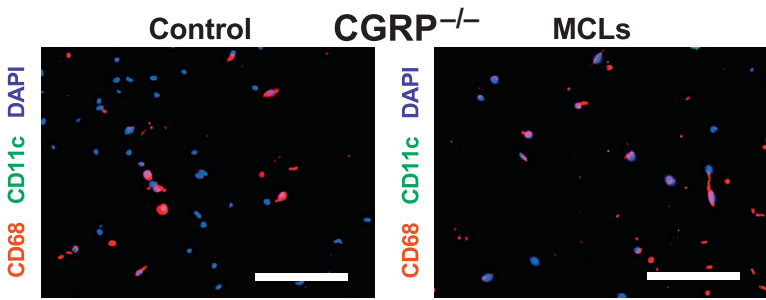

G

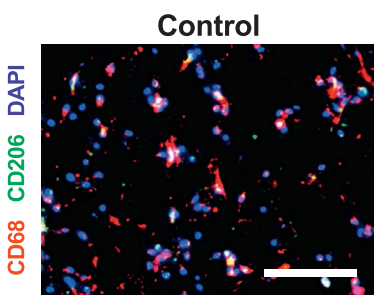

WT
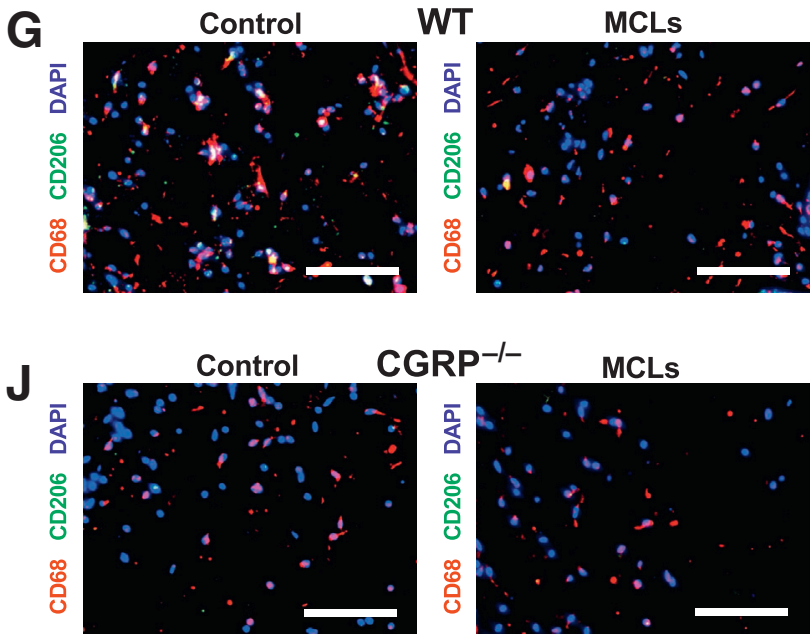
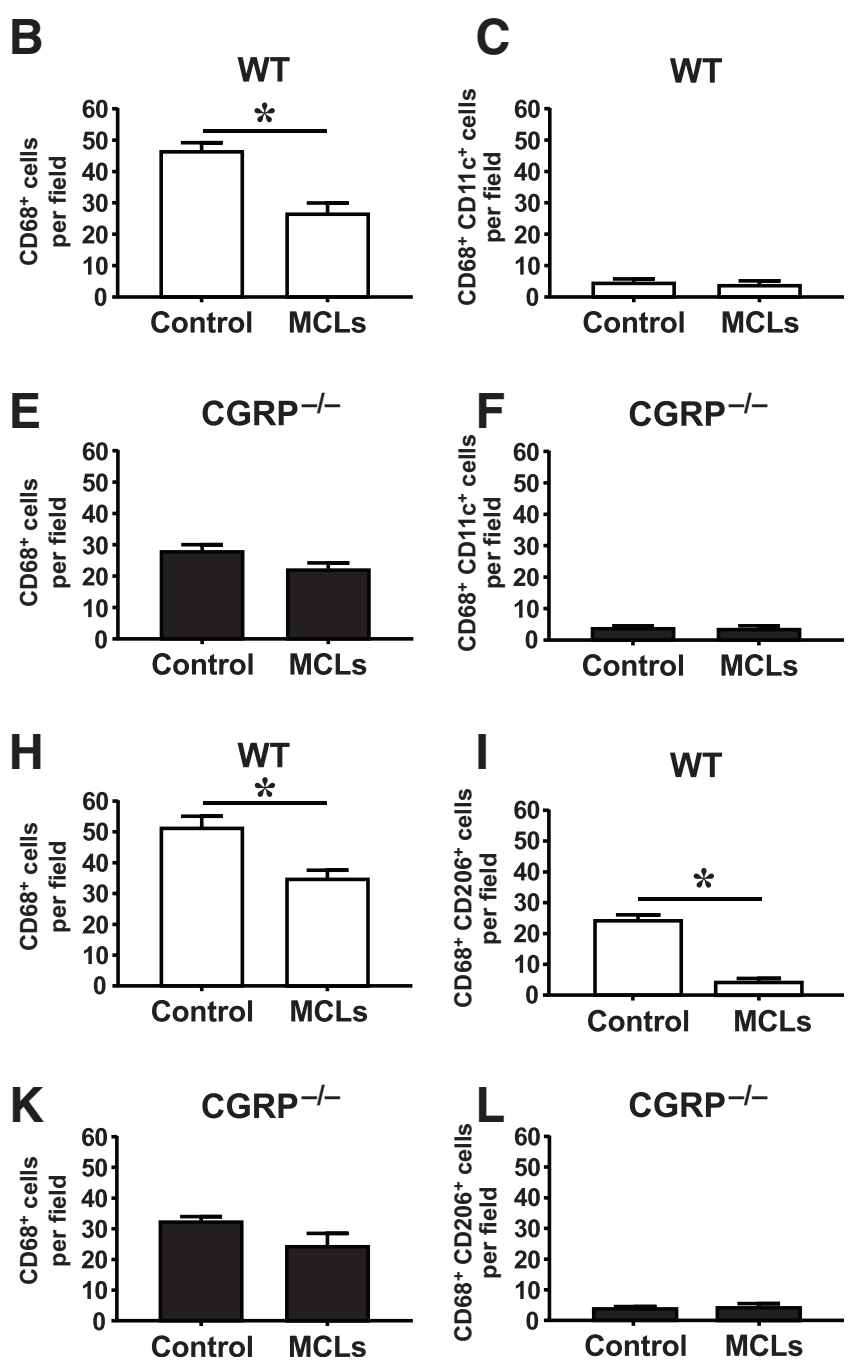

Figure 10 Mannosylated clodronate liposome (MCL) administration does not affect the numbers of M1 macrophages but decreases the numbers of M2 macrophages in wild-type (WT) mice. A and D: Immunofluorescence staining for CD68 (pan-macrophage marker) and CD11c (M1 macrophage marker) in the cross sections from the lymphedematous tails of WT (A) and calcitonin gene-related peptide knockout (CGRP ${ }^{-/}$) (D) mice on postoperative day 10 with or without MCL administration: red indicates CD68; green, CD11c; blue, DAPI. Sections were collected at tails 0.5 to $1 \mathrm{~cm}$ distal to the wounds. B and C: Numbers of $\mathrm{CD}_{68}^{+}$cells $(\mathbf{B})$ and $\mathrm{CD}_{8} 8^{+}$and $\mathrm{CD} 11 \mathrm{c}^{+}$cells $(\mathbf{C})$ in WT mice on postoperative day 10, with or without MCLs. $\mathbf{E}$ and $\mathbf{F}$ : Number of CD68 ${ }^{+}(\mathbf{E})$ and $\mathrm{CD} 68^{+}$and $\mathrm{CD}_{11 \mathrm{C}^{+}}(\mathbf{F})$ cells in $\mathrm{CGRP}^{-/-}$mice on postoperative day 10, with or without MCLs. $\mathbf{G}$ and $\mathbf{J}$ : Immunofluorescence staining for CD68 (pan-macrophage marker) and CD206 (M2 macrophage marker) in the cross sections from the lymphedematous tails of WT (G) and CGRP ${ }^{-1}$ (J) mice on postoperative day 10, with or without MCL administration: red indicates CD68; green, CD206; blue, DAPI. Sections were collected at tails 0.5 to $1 \mathrm{~cm}$ distal to the wounds. H and I: Numbers of $\mathrm{CD}^{+} 8^{+}(\mathbf{H})$ and $\mathrm{CD}^{+} 8^{+}$and $\mathrm{CD}_{206^{+}}(\mathrm{I})$ cells in WT mice on postoperative day 10, with or without MCLs. $\mathbf{K}$ and $\mathbf{L}$ : Numbers of CD68 ${ }^{+}(\mathrm{K})$ and $\mathrm{CD} 68^{+}$and

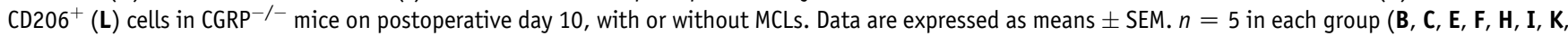
and $\mathbf{L})$. ${ }^{*} P<0.05$ (t-test). Scale bars: $100 \mu \mathrm{m}(\mathbf{A}, \mathbf{D}, \mathbf{G}$, and $\mathbf{J})$.

$\mathrm{RAMP}^{-l-}$ mice have been reported to be born normally, without any major abnormalities. However, RAMP3 ${ }^{-1-}$ mice showed more severe interstitial edema than WT mice in the tail lymphedema model. ${ }^{39}$ ADM-RAMP3 signaling likely regulates lymphatic function in adults. ${ }^{39}$ Although both $\mathrm{RAMP}^{-1-}$ and CGRP-/- mice showed severe postoperative lymphedema in the tail lymphedema model, histologic examination of the edematous tissue revealed that the conditions differed somewhat. In this study, both the number and luminal area of lymphatic capillaries were decreased in $\mathrm{CGRP}^{-1-}$ mice, whereas previously abnormal dilatation of lymphatic vessels was observed in RAMP $3^{-1-}$ mice, with no change in the number of lymphatic capillaries. The lymphatic capillary dilatation in RAMP $3^{-1-}$ mice most likely reflects disruption of lymphatic pumping. Thus, the actions of ADM and CGRP in postoperative lymphedema may not be identical: ADM-RAMP3 signaling mainly regulates the functionality of lymphatic vessels, whereas CGRP mainly regulates de novo lymphatic capillary formation. Note that, individually, neither ADM nor CGRP could reverse the lymphedema. In this study, for example, compensatory up-regulation of ADM was detected in the 
A

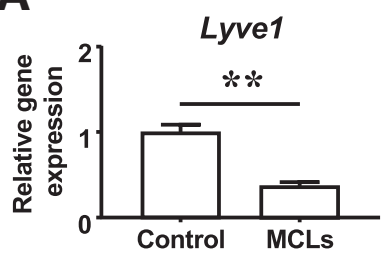

B

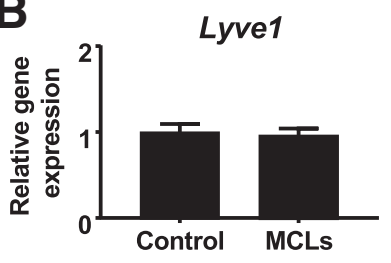

Prox1

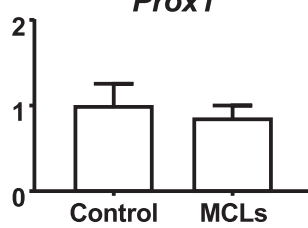

Control MCLs

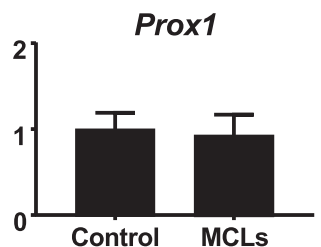

WT

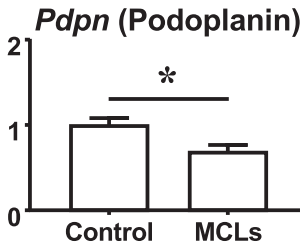

CGRP $^{-1-}$

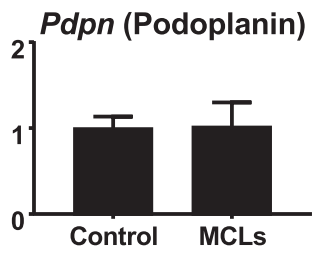

WT
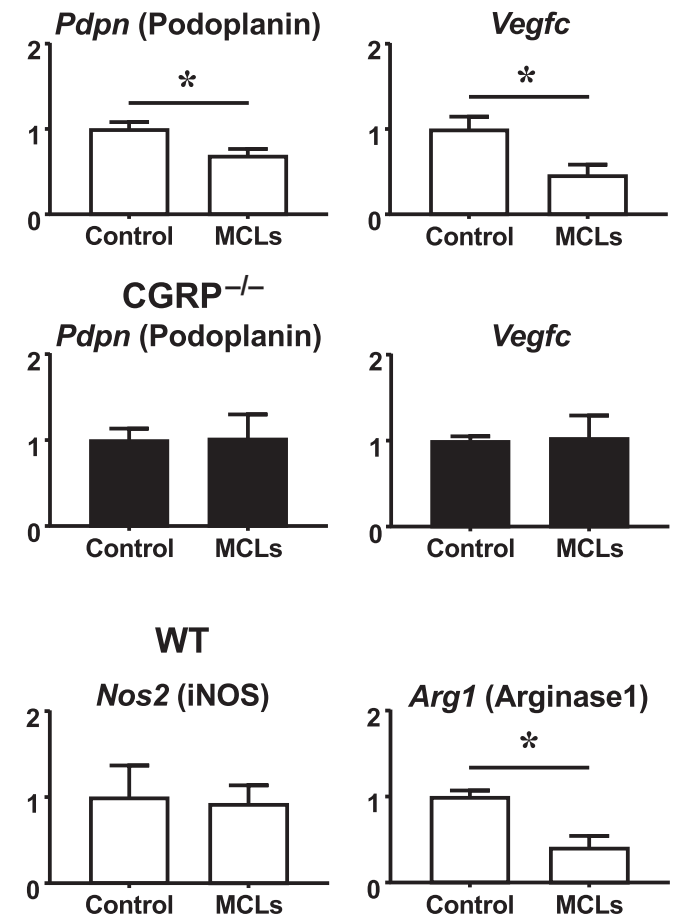

D

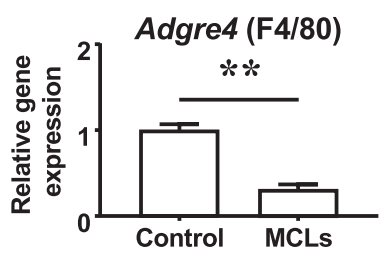

\section{CGRP $^{-/-}$}
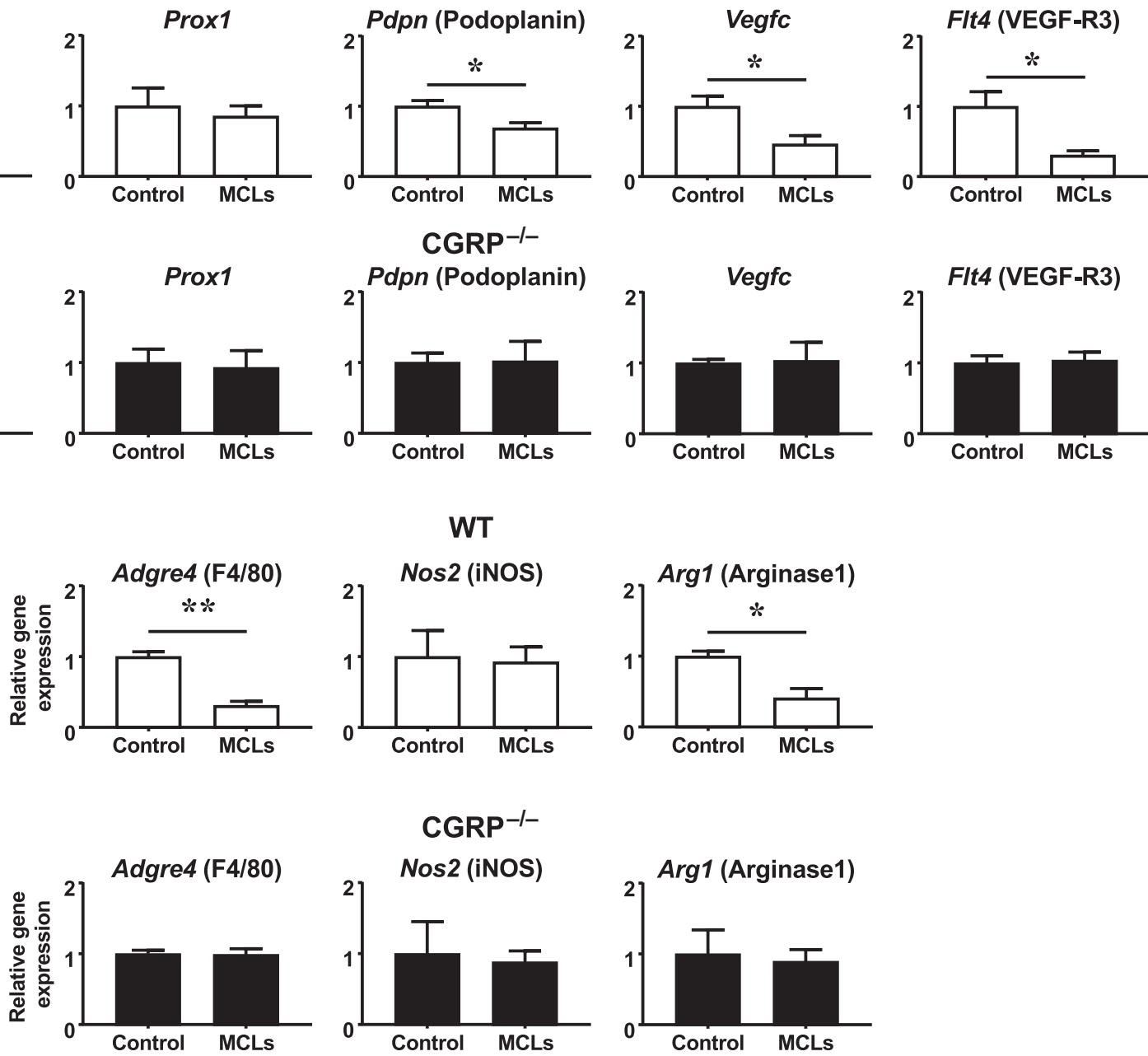

Figure 11 Effect of mannosylated clodronate liposome (MCL) administration on expression of lymphatic capillary formation-related genes and macrophage markers in postoperative lymphedematous tails. A and B: Expression levels of mRNAs encoding lymphatic vessel endothelial hyaluronan receptor 1 (LYVE-1), PROX1, Podoplanin, vascular endothelial growth factor (VEGF)-C, and vascular endothelial growth factor receptor (VEGFR)-3 in the lymphedematous tails of wild-type (WT) (A) and calcitonin gene-related peptide knockout (CGRP ${ }^{-1-}$ ) (B) mice on postoperative day 10, with or without MCL administration. Tissues were collected from a tail region 1 to $2 \mathrm{~cm}$ distal to the wounds, and mRNA expression levels were determined with real-time RT-PCR. Data from WT mice without MCLs (A) and CGPR ${ }^{-1-}$ mice without MCL (B) were assigned a value of 1. C and D: Expression levels of mRNAs encoding F4/80 (pan-macrophage maker), inducible nitric oxide synthase (iNOS) (M1 macrophage maker), and Arginase1 (M2 macrophage maker) in the postoperative lymphedematous tails of WT (C) and $\mathrm{CGRP}^{-1-}$ (D) mice on postoperative day 10, with or without MCL administration. Tissues were collected from a tail region 1 to $2 \mathrm{~cm}$ distal to the wounds, and mRNA expression levels were determined by real-time RT-PCR. Data from the WT mice without MCLs (C) and CGRP ${ }^{-/-}$mice without MCLs (D) were assigned a value of 1 . Data are expressed as means \pm SEM. $n=3$ in each group (A-D). ${ }^{* *} P<0.01,{ }^{*} P<0.05$ ( $t$-test).

postoperative tissue of $\mathrm{CGRP}^{-/-}$mice; nevertheless, postoperative edema was more severe in $\mathrm{CGRP}^{-1-}$ mice than in WT mice, indicating that ADM cannot be used to replace CGRP and vice versa. Therefore, both ADM and CGRP are necessary for proper lymphatic system restoration after surgery.

With the use of real-time PCR analysis, lymphatic capillary formation-related factors were found to be upregulated after the operation. Among them, VEGFR-3 and its ligand VEGF-C have been identified as important regulators of lymphatic capillary formation, and the therapeutic potential of VEGF-C has been demonstrated. ${ }^{40}$ In CGRP $^{-1-}$ mice, expression levels of both Flt4 (VEGFR-3) and Vegfc were significantly lower than in WT mice, demonstrating that endogenous CGRP is necessary for the proper induction of lymphatic capillary formation-related factors.

An accumulation of macrophages within postoperative lymphedematous tissue was also detected. Macrophages are recruited and accumulate under various inflammatory conditions. Recent studies have shown that macrophages are a major source of VEGF-C, which plays a pivotal role in activating the proliferation of lymphatic endothelial cells. ${ }^{41}$ In this study, the numbers of macrophages within postoperative lymphedematous tissue were significantly lower in $\mathrm{CGRP}^{-1-}$ mice than in WT mice. Moreover, there was a selective reduction in the numbers of M2 macrophages in 


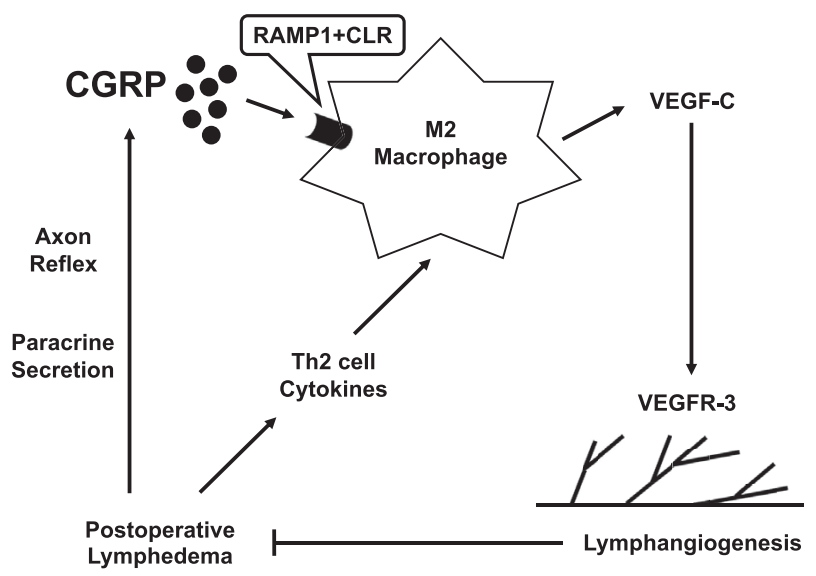

Figure 12 Schematic diagram of the mechanism by which lymphedema is exacerbated in calcitonin gene-related peptide knockout $\left(\mathrm{CGRP}^{-/-}\right)$ mice. The operative procedure inevitably causes tissue damages and lymphedema. In wild-type (WT) mice, CGRP is secreted from axons via the axonal reflex or other cells as a paracrine factor. Locally secreted CGRP acts on M2 macrophages. Once activated, M2 macrophages produce vascular endothelial growth factor (VEGF)-C, which binds to vascular endothelial growth factor receptor (VEGFR)-3 of lymphatic endothelial cells to promote lymphatic capillary formation. Induction of lymphatic capillary formation promotes drainage from the damaged tissue and ameliorates the postoperative lymphedema. In CGRP ${ }^{-/-}$mice, the CGRP deficiency suppresses M2 macrophage activation and lymphatic capillary formation, thereby worsening the edema. CLR, calcitonin receptor-like receptor; RAMP1, receptor activity-modifying protein 1 ; Th2, T helper type 2 .

$\mathrm{CGRP}^{-1-}$ mice. Classically activated macrophages with what is known as the M1 phenotype provoke secretion of proinflammatory cytokines. ${ }^{42}$ Alternatively, activated macrophages with the M2 phenotype secrete anti-inflammatory cytokines and growth factors. ${ }^{43}$ In this study, Il6 expression was significantly elevated in $\mathrm{CGRP}^{-1-}$ mice on day 10 after surgery. Because IL- 6 was recently described as an important determinant of M2 macrophage polarization, ${ }^{44}$ the elevation of IL-6 may be a compensatory response to the impaired M2 macrophage induction in $\mathrm{CGRP}^{-1-}$ mice.

Selectively depleting M2 macrophages with the use of MCLs in the postoperative lymphedema model exacerbated postoperative lymphedema in WT mice, but had little effect in $\mathrm{CGRP}^{-1-}$ mice. Histologic analysis showed that MCL administration reduced both the accumulation of M2 macrophages and lymphatic capillary formation in WT mice. Numbers of M2 macrophages were already diminished in $\mathrm{CGRP}^{-1-}$ mice without MCLs, and MCL administration did not reduce it further. Taken together, these findings clearly show that CGRP promotes M2 macrophage accumulation and lymphatic capillary formation and that the increased severity of lymphedema seen in $\mathrm{CGRP}^{-1-}$ mice is related to a reduction in M2 macrophages. M2 macrophages are known to be activated by the T helper type 2 type cytokines IL-4 and IL-13. ${ }^{45}$ Although the relationship between CGRP and $\mathrm{T}$ helper type 2 cytokines remains unclear, CGRP could be a novel agent able to activate M2 macrophages.
The CLR-RAMP1 receptor complex has higher affinity for CGRP. During the preparation of this article, Mishima et $\mathrm{al}^{24}$ reported a similar tail lymphedema model that used RAMP $1^{-/}$mice. They found that, compared with WT mice, lymphedema in the tails in $\mathrm{RAMP}^{-1-}$ mice was more sustained, with suppressed lymphatic capillary formation and reduced expression of VEGF-C and VEGFR-3. This result together with that of the present findings further supports the idea that CGRP-RAMP1 signaling plays a critical role in mediating lymphatic capillary formation. However, a difference between the two studies is that Mishima et $\mathrm{al}^{24}$ removed a larger section of tissue and evaluated the effect later ( 6 weeks after surgery) than done in this study. They observed maximal tail thickening at 2 to 3 weeks, and edema that was significantly more severe in $\mathrm{RAMP}^{-1-}$ mice than in WT mice continued for up to 6 weeks. By contrast, peak swelling was observed on days 10 to 12 , and the difference between WT and $\mathrm{CGRP}^{-1-}$ mice was transient. As it progresses, postoperative lymphedema becomes associated with fibrosis. Indeed, a recent study showed that fibrosis induced by chronic inflammation plays a key role in the pathophysiology of the later stages of postoperative lymphedema. ${ }^{46}$ This reflects the fact that lymphatic injury activates chronic immune responses that promote fibrosis and decrease lymphatic pumping. Attention should therefore be paid to whether the observed tail thickening is mainly caused by impaired lymphatic capillary formation or enhanced fibrosis.

Figure 12 summarizes a proposed mechanism underlying the exacerbation of lymphedema in $\mathrm{CGRP}^{-/-}$mice. The operative procedure inevitably causes tissue damage. In WT mice, CGRP is secreted from axons via the axonal reflex or from other cells as a paracrine factor. Locally secreted CGRP mediates M2 macrophage accumulation and activation. M2 macrophages then produce VEGF-C, which binds to VEGFR-3 on lymphatic endothelial cells to promote lymphatic capillary formation. Induction of lymphatic capillary formation promotes drainage from the damaged tissue and ameliorates the postoperative lymphedema. In $\mathrm{CGRP}^{-1-}$ mice, CGRP deficiency suppresses M2 macrophage activation and lymphatic capillary formation, thereby exacerbating the edema.

In a preliminary study, it was observed that CGRPadministration to $\mathrm{CGRP}^{-1-}$ mice ameliorated postoperative lymphedema. Although this finding supports the idea that exogenously administered CGRP would be beneficial, the proper dosage, route of administration, and effect duration remain to be determined. Further studies around the optimal usage of CGRP could help to define its therapeutic value for the treatment of postoperative lymphedema.

\section{Supplemental Data}

Supplemental material for this article can be found at http://doi.org/10.1016/j.ajpath.2019.08.011. 


\section{References}

1. Maegawa J, Yabuki Y, Tomoeda H, Hosono M, Yasumura K: Outcomes of lymphaticovenous side-to-end anastomosis in peripheral lymphedema. J Vasc Surg 2012, 55:753-760

2. Demirtas Y, Ozturk N, Yapici O, Topalan M: Supermicrosurgical lymphaticovenular anastomosis and lymphaticovenous implantation for treatment of unilateral lower extremity lymphedema. Microsurgery 2009, 29:609-618

3. Rosenfeld MG, Mermod JJ, Amara SG, Swanson LW, Sawchenko PE, Rivier J, Vale WW, Evans RM: Production of a novel neuropeptide encoded by the calcitonin gene via tissue-specific RNA processing. Nature 1983, 304:129-135

4. Eftekhari S, Salvatore CA, Johansson S, Chen TB, Zeng Z, Edvinsson L: Localization of CGRP, CGRP receptor, PACAP and glutamate in trigeminal ganglion. Relation to the blood-brain barrier. Brain Res 2015, 1600:93-109

5. Kruger L, Mantyh PW, Sternini C, Brecha NC, Mantyh CR: Calcitonin gene-related peptide (CGRP) in the rat central nervous system: patterns of immunoreactivity and receptor binding sites. Brain Res 1988, 463:223-244

6. Kawasaki H, Takasaki K, Saito A, Goto K: Calcitonin gene-related peptide acts as a novel vasodilator neurotransmitter in mesenteric resistance vessels of the rat. Nature 1988, 335:164-167

7. Uddman R, Edvinsson L, Ekblad E, Hakanson R, Sundler F: Calcitonin gene-related peptide (CGRP): perivascular distribution and vasodilatory effects. Regul Pept 1986, 15:1-23

8. Villarreal D, Reams G, Freeman R: Calcitonin gene-related peptide and the kidney. Curr Opin Nephrol Hypertens 1994, 3:453-458

9. Matteoli M, Haimann C, Torri-Tarelli F, Polak JM, Ceccarelli B, De Camilli P: Differential effect of alpha-latrotoxin on exocytosis from small synaptic vesicles and from large dense-core vesicles containing calcitonin gene-related peptide at the frog neuromuscular junction. Proc Natl Acad Sci U S A 1988, 85:7366-7370

10. Rezaeian AH, Isokane $T$, Nishibori $M$, Chiba M, Hiraiwa N, Yoshizawa M, Yasue H: AlphaCGRP and betaCGRP transcript amount in mouse tissues of various developmental stages and their tissue expression sites. Brain Dev 2009, 31:682-693

11. Ramos-Romero ML, Sobrino-Mejia FE: [Calcitonin gene-related peptide: a key player neuropeptide in migraine] Spanish. Rev Neurol 2016, 63:460-468

12. Chai W, Mehrotra S, Jan Danser AH, Schoemaker RG: The role of calcitonin gene-related peptide (CGRP) in ischemic preconditioning in isolated rat hearts. Eur J Pharmacol 2006, 531:246-253

13. Li J, Levick SP, DiPette DJ, Janicki JS, Supowit SC: Alpha-calcitonin gene-related peptide is protective against pressure overload-induced heart failure. Regul Pept 2013, 185:20-28

14. Mishima T, Ito $\mathrm{Y}$, Hosono $\mathrm{K}$, Tamura $\mathrm{Y}$, Uchida $\mathrm{Y}$, Hirata $\mathrm{M}$, Suzsuki T, Amano H, Kato S, Kurihara Y, Kurihara H, Hayashi I, Watanabe M, Majima M: Calcitonin gene-related peptide facilitates revascularization during hindlimb ischemia in mice. Am J Physiol Heart Circ Physiol 2011, 300:H431-H439

15. Zheng S, Li W, Xu M, Bai X, Zhou Z, Han J, Shyy JY, Wang X: Calcitonin gene-related peptide promotes angiogenesis via AMPactivated protein kinase. Am J Physiol Cell Physiol 2010, 299: C1485-C1492

16. Just S, Arndt K, Doods H: The role of CGRP and nicotinic receptors in centrally evoked facial blood flow changes. Neurosci Lett 2005, 381:120-124

17. Wang Z, Martorell BC, Walchli T, Vogel O, Fischer J, Born W, Vogel J: Calcitonin gene-related peptide (CGRP) receptors are important to maintain cerebrovascular reactivity in chronic hypertension. PLoS One 2015, 10:e0123697

18. Fernandes ES, Schmidhuber SM, Brain SD: Sensory-nerve-derived neuropeptides: possible therapeutic targets. Handb Exp Pharmacol 2009, (194):393-416
19. Permpoonputtana K, Porter JE, Govitrapong P: Calcitonin generelated peptide mediates an inflammatory response in Schwann cells via cAMP-dependent ERK signaling cascade. Life Sci 2016, 144:19-25

20. McLatchie LM, Fraser NJ, Main MJ, Wise A, Brown J, Thompson N, Solari R, Lee MG, Foord SM: RAMPs regulate the transport and ligand specificity of the calcitonin-receptor-like receptor. Nature 1998, 393:333-339

21. Kurashige C, Hosono K, Matsuda H, Tsujikawa K, Okamoto H, Majima M: Roles of receptor activity-modifying protein 1 in angiogenesis and lymphangiogenesis during skin wound healing in mice. FASEB J 2014, 28:1237-1247

22. Oh-hashi Y, Shindo T, Kurihara Y, Imai T, Wang Y, Morita H, Imai Y, Kayaba Y, Nishimatsu H, Suematsu Y, Hirata Y, Yazaki Y, Nagai R, Kuwaki T, Kurihara H: Elevated sympathetic nervous activity in mice deficient in alphaCGRP. Circ Res 2001, 89:983-990

23. Jin D, Harada K, Ohnishi S, Yamahara K, Kangawa K, Nagaya N: Adrenomedullin induces lymphangiogenesis and ameliorates secondary lymphoedema. Cardiovasc Res 2008, 80:339-345

24. Mishima T, Ito Y, Nishizawa N, Amano H, Tsujikawa K, Miyaji K, Watanabe M, Majima M: RAMP1 signaling improves lymphedema and promotes lymphangiogenesis in mice. J Surg Res 2017, 219: $50-60$

25. Kashiwagi S, Hosono K, Suzuki T, Takeda A, Uchinuma E, Majima M: Role of COX-2 in lymphangiogenesis and restoration of lymphatic flow in secondary lymphedema. Lab Invest 2011, 91: $1314-1325$

26. Hagendoorn J, Padera TP, Kashiwagi S, Isaka N, Noda F, Lin MI, Huang PL, Sessa WC, Fukumura D, Jain RK: Endothelial nitric oxide synthase regulates microlymphatic flow via collecting lymphatics. Circ Res 2004, 95:204-209

27. Pytowski B, Goldman J, Persaud K, Wu Y, Witte L, Hicklin DJ, Skobe M, Boardman KC, Swartz MA: Complete and specific inhibition of adult lymphatic regeneration by a novel VEGFR-3 neutralizing antibody. J Natl Cancer Inst 2005, 97:14-21

28. Swartz MA, Berk DA, Jain RK: Transport in lymphatic capillaries. I. Macroscopic measurements using residence time distribution theory. Am J Physiol 1996, 270:H324-H329

29. Zhou Y, Yoshida S, Nakao S, Yoshimura T, Kobayashi Y, Nakama T, Kubo Y, Miyawaki K, Yamaguchi M, Ishikawa K, Oshima Y, Akashi K, Ishibashi T: M2 macrophages enhance pathological neovascularization in the mouse model of oxygen-induced retinopathy. Invest Ophthalmol Vis Sci 2015, 56:4767-4777

30. Zhai L, Sakurai T, Kamiyoshi A, Ichikawa-Shindo Y, Kawate H, Tanaka M, Xian X, Hirabayashi K, Dai K, Cui N, Tanimura K, Liu T, Wei Y, Tanaka M, Tomiyama H, Yamauchi A, Igarashi K, Shindo T: Endogenous calcitonin gene-related peptide suppresses ischemic brain injuries and progression of cognitive decline. J Hypertens 2018, $36: 876-891$

31. Venero Galanternik M, Stratman AN, Jung HM, Butler MG, Weinstein BM: Building the drains: the lymphatic vasculature in health and disease. Wiley Interdiscip Rev Dev Biol 2016, 5:689-710

32. Hartiala P, Saarikko AM: Lymphangiogenesis and lymphangiogenic growth factors. J Reconstr Microsurg 2016, 32:10-15

33. Shindo T, Kurihara Y, Nishimatsu H, Moriyama N, Kakoki M, Wang Y, Imai Y, Ebihara A, Kuwaki T, Ju KH, Minamino N, Kangawa K, Ishikawa T, Fukuda M, Akimoto Y, Kawakami H, Imai T, Morita H, Yazaki Y, Nagai R, Hirata Y, Kurihara H: Vascular abnormalities and elevated blood pressure in mice lacking adrenomedullin gene. Circulation 2001, 104:1964-1971

34. Caron KM, Smithies O: Extreme hydrops fetalis and cardiovascular abnormalities in mice lacking a functional Adrenomedullin gene. Proc Natl Acad Sci U S A 2001, 98:615-619

35. Iimuro S, Shindo T, Moriyama N, Amaki T, Niu P, Takeda N, Iwata $\mathrm{H}$, Zhang $\mathrm{Y}$, Ebihara A, Nagai R: Angiogenic effects of adrenomedullin in ischemia and tumor growth. Circ Res 2004, 95: $415-423$ 
36. Nikitenko LL, Shimosawa $\mathrm{T}$, Henderson $\mathrm{S}$, Makinen $\mathrm{T}$, Shimosawa H, Qureshi U, Pedley RB, Rees MC, Fujita T, Boshoff C: Adrenomedullin haploinsufficiency predisposes to secondary lymphedema. J Invest Dermatol 2013, 133:1768-1776

37. Hoopes SL, Willcockson HH, Caron KM: Characteristics of multiorgan lymphangiectasia resulting from temporal deletion of calcitonin receptor-like receptor in adult mice. PLoS One 2012, 7: e45261

38. Ichikawa-Shindo Y, Sakurai T, Kamiyoshi A, Kawate H, Iinuma N, Yoshizawa T, Koyama T, Fukuchi J, Iimuro S, Moriyama $\mathrm{N}$, Kawakami H, Murata T, Kangawa K, Nagai R, Shindo T: The GPCR modulator protein RAMP2 is essential for angiogenesis and vascular integrity. J Clin Invest 2008, 118:29-39

39. Yamauchi A, Sakurai T, Kamiyoshi A, Ichikawa-Shindo $\mathrm{Y}$, Kawate H, Igarashi K, Toriyama Y, Tanaka M, Liu T, Xian X, Imai A, Zhai L, Owa S, Arai T, Shindo T: Functional differentiation of RAMP2 and RAMP3 in their regulation of the vascular system. J Mol Cell Cardiol 2014, 77:73-85

40. Szuba A, Skobe M, Karkkainen MJ, Shin WS, Beynet DP, Rockson NB, Dakhil N, Spilman S, Goris ML, Strauss HW, Quertermous T, Alitalo K, Rockson SG: Therapeutic lymphangiogenesis with human recombinant VEGF-C. FASEB J 2002, 16:1985-1987

41. Corliss BA, Azimi MS, Munson JM, Peirce SM, Murfee WL: Macrophages: an inflammatory link between angiogenesis and lymphangiogenesis. Microcirculation 2016, 23:95-121

42. Martinez FO, Gordon S: The M1 and M2 paradigm of macrophage activation: time for reassessment. F1000Prime Rep 2014, 6:13

43. Roszer T: Understanding the mysterious M2 macrophage through activation markers and effector mechanisms. Mediators Inflamm 2015, 2015:816460

44. Mauer J, Chaurasia B, Goldau J, Vogt MC, Ruud J, Nguyen KD, Theurich S, Hausen AC, Schmitz J, Bronneke HS, Estevez E, Allen TL, Mesaros A, Partridge L, Febbraio MA, Chawla A, Wunderlich FT, Bruning JC: Signaling by IL-6 promotes alternative activation of macrophages to limit endotoxemia and obesityassociated resistance to insulin. Nat Immunol 2014, 15:423-430

45. Murray PJ, Wynn TA: Protective and pathogenic functions of macrophage subsets. Nat Rev Immunol 2011, 11:723-737

46. Kataru RP, Wiser I, Baik JE, Park HJ, Rehal S, Shin JY, Mehrara BJ: Fibrosis and secondary lymphedema: chicken or egg? Transl Res 2019, 209:68-76 